Working Paper 12-18-(12)

Statistics and Econometrics Series

June, 2012
Departamento de Estadística

Universidad Carlos III de Madrid

Calle Madrid, 126 28903 Getafe (Spain)

Fax (34) 91 624-98-49

\title{
PORTFOLIO SELECTION THROUGH AN EXTREMALITY STOCHASTIC ORDER
}

\author{
Henry Laniado ${ }^{\mathrm{a}}$, Rosa E. Lillo ${ }^{\mathrm{b}}$, Franco Pellerey ${ }^{\mathrm{c}}$ and Juan Romo ${ }^{\mathrm{b}}$ \\ aDepartamento de Estadística, Universidad Carlos III de Madrid, 28911, Leganés, Spain \\ b Departamento de Estadística, Universidad Carlos III de Madrid, 28903, Getafe, Spain \\ cDipartimento di Matematica, Politecnico di Torino, 10129, Torino, Italy
}

\begin{abstract}
In this paper we introduce a new multivariate stochastic order that compares random vectors in a direction which is determined by a unit vector, generalizing previous upper and lower orthant order. The main properties of this new order, together with its relationships with other multivariate stochastic orders, are investigated and, we present some examples of application in the determination of optimal allocations of wealth among risks in single period portfolio problems.
\end{abstract}

Keywords: Portfolio selection, extremality, upper orthant 2010 MSC: 60E15, 62P05, 91G10

Email addresses: henry.laniado@uc3m.es (Henry Laniado), rosaelvira.lillo@uc3m.es (Rosa E. Lillo), franco.pellerey@polito.it (Franco Pellerey), juan.romo@uc3m.es (Juan Romo) 


\title{
Portfolio selection through an extremality stochastic order
}

\author{
Henry Laniado ${ }^{\mathrm{a}}$, Rosa E. Lillo ${ }^{\mathrm{b}}$, Franco Pellerey ${ }^{\mathrm{c}}$, Juan Romo ${ }^{\mathrm{b}}$ \\ ${ }^{a}$ Departamento de Estadística, Universidad Carlos III de Madrid, 28911, Leganés, Spain \\ ${ }^{b}$ Departamento de Estadística, Universidad Carlos III de Madrid, 28903, Getafe, Spain \\ ${ }^{c}$ Dipartimento di Matematica, Politecnico di Torino, 10129, Torino, Italy
}

\begin{abstract}
In this paper we introduce a new multivariate stochastic order that compares random vectors in a direction which is determined by a unit vector, generalizing previous upper and lower orthant order. The main properties of this new order, together with its relationships with other multivariate stochastic orders, are investigated and, we present some examples of application in the determination of optimal allocations of wealth among risks in single period portfolio problems.
\end{abstract}

Keywords: Portfolio selection, Extremality, Upper orthant

2010 MSC: 60E15, 62P05, 91G10

\section{Introduction}

One of the most relevant tools in risk evaluations of portfolios of hedge funds was introduced by Markowitz [16]. In his approach, risky investments comparisons are carried out through means and variances of the prospects:

Email addresses: henry.laniado@uc3m.es (Henry Laniado), rosaelvira.1illo@uc3m.es (Rosa E. Lillo), franco.pellerey@polito.it (Franco Pellerey), juan.romo@uc3m.es (Juan Romo) 
given a random vector of risky assets $\mathbf{X}=\left(X_{1}, \ldots, X_{n}\right)^{\prime}$ and a real vector $\mathbf{w}=\left(\omega_{1}, \ldots, \omega_{n}\right)^{\prime}$ describing the allocation of wealth, the risk averse decision maker assigns to the portfolio $Z_{\mathbf{X}}=\mathbf{w}^{\prime} \mathbf{X}$ the utility $U\left(Z_{\mathbf{X}}\right)=$ $E\left(Z_{\mathbf{X}}\right)-\alpha \operatorname{Var}\left(Z_{\mathbf{X}}\right)$, where $\alpha>0$ is the degree of risk aversion, and choose among portfolios maximizing the utility $U\left(Z_{\mathbf{X}}\right)$. Markowitz model has some drawbacks; for instance, it is not consistent with respect to the usual stochastic order (see Müller and Stoyan [18]), where the consistency is the monotonicity of a utility function or of a risk measure with respect to some stochastic order (see Bauerle and Müller [2] and references therein). In fact, starting from the assumption that utility functions are increasing and concave, which is common in economic theory, consistency means that stochastic comparisons between two different vectors $\mathbf{X}$ and $\mathbf{Y}$ of risky assets implies comparisons between the utilities $E U\left(Z_{\mathbf{X}}\right)$ and $E U\left(Z_{\mathbf{Y}}\right)$ for the same vector of allocations,. The aim of this paper is to introduce a new multivariate stochastic order that may be useful in finding out new guidelines for allocation of risks in static portfolios.

Comparisons among random variables and vectors in different stochastic ways have been extensively considered during the last thirty years. Applications of these stochastic orderings have been provided in several disciplines, from economic theory to reliability and queueing theory (see, e.g., Barlow and Proschan [1], Stoyan [23], Shaked and Shanthikumar [24], Denuit et al. [3]). Among the stochastic orders defined and studied in the literature, most of them deal with comparisons between random vectors, like the multivariate usual stochastic order or the multivariate dispersion orders, with applications in decision making in multiple output scenarios. 
In this paper we introduce a new multivariate stochastic order, called extremality order, that is a generalization of the upper and lower orthant order discussed in Shaked and Shanthikumar [24] and Marshall and Olkin [17], and that, unlike these two orders, allows comparisons of random vectors in different directions, determined by a unit vector. Essentially, it is based on rotation of the non-negative orthant in a direction given, obtaining a cone, which is isomorph to the non-negative orthant.

The extremality order is based on the multivariate data ordering introduced in Laniado et al. [13], where an extremality measure was defined to find multivariate extremes from a directional approach. Inspired on the extremality directional data ordering, we propose in this paper a probabilistic comparison between multivariate random vectors based on the probability assigned on some extremes sets on a given direction. We show in the paper that, in portfolio comparisons, other directions can be more interesting than those used to define the upper and lower orthant orders.

The paper is organized as follows. Some preliminaries are described in Section 2 , while the properties of the extremality order, and its relationships with other multivariate stochastic orders (in particular, with the upper orthant and lower orthant orders), are described in Section 3. A list of its applications in portfolio theory are provided in Sections 4 and 5. Finally, in Section 6 we summarize the main conclusions.

\section{Preliminaries}

For ease of reference, first we briefly recall some notation that will be used throughout the paper. Random vectors taking values in $\mathbb{R}^{n}$ will be 
considered, unless otherwise stated. The space $\mathbb{R}^{n}$ is endowed with the usual componentwise partial order, which is defined as follows: given two vectors $\mathbf{x}=\left(x_{1}, \cdots, x_{n}\right)$ and $\mathbf{y}=\left(y_{1}, \cdots, y_{n}\right)$ in $\mathbb{R}^{n}$, then $\mathbf{x} \leq \mathbf{y}$ if $x_{i} \leq y_{i}$ for $i=1, \cdots, n$. A function $\phi: \mathbb{R}^{n} \longrightarrow \mathbb{R}^{n}$ is said to be an increasing function when $\phi(\mathbf{x}) \leq \phi(\mathbf{y})$ for $\mathbf{x} \leq \mathbf{y}$. Throughout the paper the terms 'increasing' and 'decreasing' stand for 'non-decreasing' and 'non-increasing', respectively. Moreover, we shall adopt the following notations: for any random variable $Z$ we shall denote its distribution function by $F_{Z}(x)=P(Z \leq x)$ and its survival function by $\bar{F}_{Z}(x)=P(Z>x)$; the notation $={ }_{s t}$ stands for the equality in law; the notation $u \wedge v$ and $u \vee v$ stand for $\min \{u, v\}$ and $\max \{u, v\}$, respectively.

Concerning the stochastic comparisons, we first provide the definition of the orders usually considered in the univariate setting.

Definition 2.1. Given two random variables $X$ and $Y$ we say that $X$ is smaller than $Y$ in the usual stochastic order [convex order, increasing convex order, increasing concave order] (denoted by $X \leq_{s t}\left[\leq_{c x}, \leq_{i c x}, \leq_{i c v}\right] Y$ ) if and only if $E[\phi(X)] \leq E[\phi(Y)]$ for all increasing [convex, increasing convex, increasing concave] functions $\phi$ for which the expectations exist. Moreover, we say that $X$ is smaller than $Y$ in the Laplace transform order (denoted by $\left.X \leq_{L t} Y\right)$ whenever $\phi(t)=-e^{-a t}$ where $a$ is any positive number.

It should be recalled that in economics, where comparisons among expected utilities are commonly considered, the usual stochastic order and the increasing concave order are referred as First order Stochastic Dominance (FSD) and Second order Stochastic Dominance (SSD), respectively. 
In the multivariate setting, the following stochastic orders have been defined as multivariate generalization of the usual stochastic order.

Definition 2.2. Given two random vectors $\mathbf{X}$ and $\mathbf{Y}, \mathbf{X}$ is said to be smaller than $\mathbf{Y}$ in:

(i) usual stochastic order (denoted by $\mathbf{X} \leq_{s t} \mathbf{Y}$ ) if $\mathrm{E}[\phi(\mathbf{X})] \leq \mathrm{E}[\phi(\mathbf{Y})]$ for any increasing function $\phi$ with finite expectations;

(ii) upper orthant order (denoted by $\mathbf{X} \leq_{u o} \mathbf{Y}$ ) if $\bar{F}_{\mathbf{X}}\left(x_{1}, \ldots, x_{n}\right) \leq \bar{F}_{\mathbf{Y}}\left(x_{1}, \ldots, x_{n}\right)$ for all $\mathbf{x}$, where $\bar{F}_{\mathbf{X}}, \bar{F}_{\mathbf{Y}}$ denote the survival function of $\mathbf{X}$ and $\mathbf{Y}$, respectively. (iii) lower orthant order (denoted by $\mathbf{X} \leq_{l o} \mathbf{Y}$ ) if $F_{\mathbf{X}}\left(x_{1}, \ldots, x_{n}\right) \geq F_{\mathbf{Y}}\left(x_{1}, \ldots, x_{n}\right.$ ) for all $\mathbf{x}$, where $F_{\mathbf{X}}, F_{\mathbf{Y}}$ denote the distribution function of $\mathbf{X}$ and $\mathbf{Y}$, respectively.

It is easy to verify that both the upper orthant order and the lower orthant order are implied by the usual stochastic order. The following two multivariate stochastic orders have been defined to compare the strength of dependence between the components of vectors in the same Fréchet class (see Lehmann [14]). Recall that a function $\phi: \mathbb{R}^{n} \rightarrow \mathbb{R}$ is said to be supermodular if $\phi(\boldsymbol{x} \vee \boldsymbol{y})+\phi(\boldsymbol{x} \wedge \boldsymbol{y}) \geq \phi(\boldsymbol{x})+\phi(\boldsymbol{y})$ for all $\boldsymbol{x}, \boldsymbol{y} \in \mathbb{R}^{n}$.

Definition 2.3. Given two random vectors $\mathbf{X}$ and $\mathbf{Y}$ having the same marginal distributions, i.e., such that $X_{i}={ }_{s t} Y_{i}$ for all $i=1, \ldots, n, \mathbf{X}$ is said to be smaller than $\mathbf{Y}$ in:

(i) positive quadrant dependence order (denoted by $\mathbf{X} \leq_{P Q D} \mathbf{Y}$ ) if $\mathbf{X} \leq_{u_{o}} \mathbf{Y}$, or, equivalently, if $\mathbf{X} \geq_{l o} \mathbf{Y}$;

(ii) supermodular order (denoted by $\mathbf{X} \leq_{s m} \mathbf{Y}$ ) if $E[\phi(\mathbf{X})] \leq E[\phi(\mathbf{Y})]$ for any supermodular function $\phi$ such that the expectations exist.

It should be pointed out that the positive quadrant dependence order 
and the supermodular order are equivalent for dimension two, while this is not true anymore in higher dimensions. Further details, properties and applications of all the stochastic orders defined above may be found, for example, in Müller and Stoyan [18], Shaked and Shanthikumar [24], or Denuit et al. [3].

\section{Extremality order}

The extremality order, defined here, is a generalization of the upper orthant and lower orthant orders, and allows for comparison of random vectors based on directions specified by a unit vector. Given $\mathbf{u} \in \mathbb{R}^{n}$ satisfying $\|\mathbf{u}\|=1$, let $\mathcal{R}_{\mathbf{u}}$ be a rotation matrix such that

$$
\mathcal{R}_{\mathbf{u}} \mathbf{u}=\frac{1}{\sqrt{n}} \mathbf{1}
$$

where $\mathbf{1}=[1, \ldots, 1]^{\prime} \in \mathbb{R}^{n}$. We can now formulate the following definition to characterize the extremality order. From now on we assume that $\|\mathbf{u}\|=1$.

Definition 3.1. Given two random vectors $\mathbf{X}$ and $\mathbf{Y}$ in $\mathbb{R}^{n}, \mathbf{X}$ is said smaller than $\mathbf{Y}$ in extremality order in the direction $\mathbf{u}$ (denoted by $\mathbf{X} \leq_{E_{\mathbf{u}}} \mathbf{Y}$ ) if

$$
P\left[\mathcal{R}_{\mathbf{u}}(\mathbf{X}-\mathbf{t}) \geq 0\right] \leq P\left[\mathcal{R}_{\mathbf{u}}(\mathbf{Y}-\mathbf{t}) \geq 0\right], \quad \text { for all } \mathbf{t} \text { in } \mathbb{R}^{n}
$$

In words, $\mathbf{X} \leq_{E_{\mathbf{u}}} \mathbf{Y}$ means that the probability that all components jointly assume "large values in the direction of $\mathbf{u}$ " is lower for $\mathbf{X}$ than for $\mathbf{Y}$, where for "large values in the direction of a unit vector $\mathbf{u}$ " we mean that $\mathbf{y}$ is larger than $\mathbf{x}$ if $\mathcal{R}_{\mathbf{u}}(\mathbf{y}-\mathbf{x}) \geq 0$. $\leq_{E_{\mathbf{u}}}$ is based on the multivariate data ordering introduced in Laniado et al. [13], where an extremality measure was defined to find multivariate extremes from a directional approach. 
It is easy to observe that, for $\mathbf{u}=\frac{1}{\sqrt{n}} \mathbf{1}$, we have the natural componentwise order in $\mathbb{R}^{n}$. Therefore, if $\mathbf{u}=\frac{1}{\sqrt{n}} \mathbf{1}$, then

$$
\mathbf{X} \leq_{E_{\mathbf{u}}} \mathbf{Y} \Longleftrightarrow \mathbf{X} \leq_{u o} \mathbf{Y} \text { and } \mathbf{X} \leq_{E_{-\mathbf{u}}} \mathbf{Y} \Longleftrightarrow \mathbf{X} \geq_{l o} \mathbf{Y}
$$

An equivalent definition of the order can be given by using the notion of oriented sub-orthants introduced in Laniado et al. [13].

Definition 3.2. Given a unit director vector $\mathbf{u} \in \mathbb{R}^{n}$ and a vertex $\mathbf{t} \in \mathbb{R}^{n}$, the Oriented Sub-Orthant $\mathcal{C}_{\mathbf{t}}^{\mathbf{u}}$ is the convex cone

$$
\mathcal{C}_{\mathbf{t}}^{\mathbf{u}}=\left\{\mathbf{x} \in \mathbb{R}^{n} \mid \mathcal{R}_{\mathbf{u}}(\mathbf{x}-\mathbf{t}) \geq \mathbf{0}\right\}
$$

Note that if $\mathbf{u}=\frac{1}{\sqrt{n}} \mathbf{1}$ then, $\mathcal{C}_{\mathbf{t}}^{\vec{u}}=\mathbf{t}+\mathbb{R}_{+}^{n}$, and therefore in this case the extremality order is equivalent to upper orthant order (and similarly for the lower orthant order).

According to Definition 3.2 another way of writing (3.2) is thus the following:

$$
\mathbf{X} \leq_{E_{\mathbf{u}}} \mathbf{Y} \Longleftrightarrow P_{\mathbf{X}}\left(\mathcal{C}_{\mathbf{t}}^{\mathbf{u}}\right) \leq P_{\mathbf{Y}}\left(\mathcal{C}_{\mathbf{t}}^{\mathbf{u}}\right), \text { for all } \mathbf{t} \in \mathbb{R}^{n}
$$

where $P_{\mathbf{X}}$ and $P_{\mathbf{Y}}$ are the probabilities induced by the joint distribution function of $\mathbf{X}$ and $\mathbf{Y}$, respectively. Note that (3.2) also is equivalent to

$$
\mathbf{X} \leq_{E_{\mathbf{u}}} \mathbf{Y} \Longleftrightarrow E\left[I_{\mathcal{C}_{\mathbf{t}}^{\mathbf{u}}}(\mathbf{X})\right] \leq E\left[I_{\mathcal{C}_{\mathbf{t}}^{\mathbf{u}}}(\mathbf{Y})\right] \text {, for all } \mathbf{t} \in \mathbb{R}^{n}
$$

where $I_{\mathcal{C}_{\mathbf{t}}^{\mathbf{u}}}$ denotes the indicator function of $\mathcal{C}_{\mathbf{t}}^{\mathbf{u}}$. However, as shown in following Example 3.3, two random vectors can be ordered in extremality even if they are not comparable in the upper or in the lower orthant orders. 
Example 3.3. Consider two random vectors $\mathbf{X}=\left(X_{1}, X_{2}\right)$ and $\mathbf{Y}=\left(Y_{1}, Y_{2}\right)$ such that they are uniformly distributed and independent margins $X_{1} \sim$ $U(0, c), \quad X_{2} \sim U(a, b), Y_{1} \sim U(0, d), Y_{2} \sim U(0, b)$, with $b>a \geq 0 d>c \geq$ 0. Let $F_{\mathbf{X}}$ and $F_{\mathbf{Y}}$ be the joint distribution functions of $\mathbf{X}$ and $\mathbf{Y}$, respectively. We can see easily that, $\bar{F}_{\mathbf{X}}(0, a)=1, \quad \bar{F}_{\mathbf{Y}}(0, a)=\frac{b-a}{b}<1, \bar{F}_{\mathbf{X}}(c, a)=0$ and $\bar{F}_{\mathbf{Y}}(c, a)=\frac{(d-c)(b-a)}{b d}>0$. Now $F_{\mathbf{X}}(c, b)=1, \quad F_{\mathbf{Y}}(c, b)<1, \quad F_{\mathbf{X}}(c, a)=0$ and $F_{\mathbf{Y}}(c, a)=\frac{a c}{b d}>0$. Therefore, $\mathbf{X}$ and $\mathbf{Y}$ are not ordered regarding the upper orthant order and are not ordered regarding the lower orthant order. However, taking $\mathbf{u}=\frac{1}{\sqrt{2}}[1,-1]^{\prime}$ as unit vector, we have that

$$
\begin{aligned}
& P_{\mathbf{X}}\left(\mathcal{C}_{(x, y)}^{\mathbf{u}}\right) \begin{cases}1 & \text { if, } x<0, y>b \\
\frac{y-b}{b-a} & \text { if } x<0, a \leq y \leq b, \\
\frac{c-x}{c} & \text { if } 0 \leq x \leq c, y>b, \\
\left(\frac{c-x}{c}\right)\left(\frac{y-b}{b-a}\right) & \text { if } 0 \leq x \leq c, a \leq y \leq b, \\
0 & \text { otherwise. }\end{cases} \\
& P_{\mathbf{Y}}\left(\mathcal{C}_{(x, y)}^{\mathbf{u}}\right) \begin{cases}1 & \text { if } x<0, y>b, \\
\frac{y}{b} & \text { if } x<0,0 \leq y \leq b, \\
\frac{d-x}{d} & \text { if } 0 \leq x \leq d, y>b, \\
\left(\frac{d-x}{d}\right)\left(\frac{y}{b}\right) & \text { if } 0 \leq x \leq d, 0 \leq y \leq b, \\
0 & \text { otherwise. }\end{cases}
\end{aligned}
$$

Assuming $(x, y) \notin(0, c) \times(a, b)$, is straightforward to see that $P_{\mathbf{X}}\left(\mathcal{C}_{(x, y)}^{\mathbf{u}}\right) \leq$ $P_{\mathbf{Y}}\left(\mathcal{C}_{(x, y)}^{\mathbf{u}}\right)$, while for $(x, y) \in(0, c) \times(a, b)$

$$
P_{\mathbf{Y}}\left(\mathcal{C}_{(x, y)}^{\mathbf{u}}\right)-P_{\mathbf{X}}\left(\mathcal{C}_{(x, y)}^{\mathbf{u}}\right)=\left(\frac{d-x}{d}\right)\left(\frac{y}{b}\right)-\left(\frac{c-x}{c}\right)\left(\frac{y-b}{b-a}\right) \geq 0
$$


since that $\left(\frac{d-x}{d}\right) \geq\left(\frac{c-x}{c}\right)$ and $\left(\frac{y}{b}\right) \geq\left(\frac{y-b}{b-a}\right)$. Hence, $P_{\mathbf{X}}\left(\mathcal{C}_{(x, y)}^{\mathbf{u}}\right) \leq P_{\mathbf{Y}}\left(\mathcal{C}_{(x, y)}^{\mathbf{u}}\right)$ for all $(x, y) \in \mathbb{R}^{2}$, and therefore, from (3.4), $\mathbf{X} \leq_{E_{\mathbf{u}}} \mathbf{Y}$.

Some properties of the extremality order are described next. The first one expresses a relation between the extremality order and the univariate stochastic order.

Property 3.4. Let $\mathcal{R}_{\mathbf{u}}^{(r . i)}$ denotes the $i$-th row of the matrix $\mathcal{R}_{\mathbf{u}}$. If $\mathbf{X} \leq_{E_{\mathbf{u}}} \mathbf{Y}$, then $\mathcal{R}_{\mathbf{u}}^{(r . i)} \mathbf{X} \leq_{s t} \mathcal{R}_{\mathbf{u}}^{(r . i)} \mathbf{Y}$ for every $i=1, \ldots, n$.

Proof. Since $\mathbf{X} \leq_{E_{\mathbf{u}}} \mathbf{Y} \Longleftrightarrow \mathcal{R}_{\mathbf{u}} \mathbf{X} \leq_{u o} \mathcal{R}_{\mathbf{u}} \mathbf{Y}$, the assertion immediately follows from Theorem 6.G.3-(c) in Shaked and Shanthikumar [24], that states that the margins of random vectors ordered in upper sense are ordered in the univariate usual stochastic order.

An immediate consequence of Property 3.4 is that, whenever $\mathbf{X}$ and $\mathbf{Y}$ have finite means,

$$
\mathbf{X} \leq_{E_{\mathbf{u}}} \mathbf{Y} \Longrightarrow \mathcal{R}_{\mathbf{u}} E[\mathbf{X}] \leq \mathcal{R}_{\mathbf{u}} E[\mathbf{Y}]
$$

Moreover, since for every vector $\mathbf{u}$ it is $\mathcal{R}_{\mathbf{u}}^{\prime} \mathcal{R}_{\mathbf{u}}=I_{n}$, and, from (3.1), $\left[\sqrt{n} \mathcal{R}_{\mathbf{u}} \mathbf{u}\right]^{\prime}=\mathbf{1}^{\prime}$, it follows

$$
\mathbf{1}^{\prime} \mathcal{R}_{\mathbf{u}} \mathbf{X}=\left[\sqrt{n} \mathcal{R}_{\mathbf{u}} \mathbf{u}\right]^{\prime} \mathcal{R}_{\mathbf{u}} \mathbf{X}=\sqrt{n} \mathbf{u}^{\prime} \mathcal{R}^{\prime}{ }_{\mathbf{u}} \mathcal{R}_{\mathbf{u}} \mathbf{X}=\sqrt{n} \mathbf{u}^{\prime} \mathbf{X}
$$

Thus, again from Property 3.4,

$$
\mathbf{X} \leq_{E_{\mathbf{u}}} \mathbf{Y} \Longrightarrow \mathbf{u}^{\prime} E[\mathbf{X}] \leq \mathbf{u}^{\prime} E[\mathbf{Y}]
$$

One reason of interest in Property 3.4 is the fact that it provides a tool to compare linear combinations of random variables, a typical problem considered in portfolio theory. It is well known that, given two sets $\left\{X_{1}, \ldots, X_{n}\right\}$ and 
$\left\{Y_{1}, \ldots, Y_{n}\right\}$ of independent random variables, if $X_{i} \leq_{s t} Y_{i}$ then $\sum_{i=1}^{n} a_{i} X_{i} \leq_{s t}$ $\sum_{i=1}^{n} a_{i} Y_{i}$ whenever $a_{i} \geq 0, i=1, \ldots, n$. A generalization of this assertion was proved by Scarsini [22], who removed the assumption of independence, proving that if $\left(X_{1}, \ldots, X_{n}\right)^{\prime}$ and $\left(Y_{1}, \ldots, Y_{n}\right)^{\prime}$ have a common copula, then the stochastic order among the marginals implies the stochastic orders among the vectors and, as a corollary, the stochastic order among positive combinations of the marginals.

Since $\mathcal{R}_{\mathbf{u}}^{(r . i)} \mathbf{X}$ and $\mathcal{R}_{\mathbf{u}}^{(r . i)} \mathbf{Y}$ are linear combinations, not necessarily positive, of marginals of $\mathbf{X}$ and $\mathbf{Y}$, then Property 3.4 describes conditions to compare non-positive linear combinations of dependent random variables, as it is shown in the following example.

Example 3.5. Let $\mathbf{X}=\left(X_{1}, X_{2}\right)^{\prime}$ and $\mathbf{Y}=\left(Y_{1}, Y_{2}\right)^{\prime}$ be two normally distributed vectors having the same covariance. Assume that $\operatorname{Var}\left(X_{1}\right)+\operatorname{Var}\left(X_{2}\right)=$ $\operatorname{Var}\left(Y_{1}\right)+\operatorname{Var}\left(Y_{2}\right)$. Clearly, $\mathbf{X}$ and $\mathbf{Y}$ can have different copulas. Let $\mathbf{u}=$ $[1,0]^{\prime}$ be the unit vector, so that the rotation matrix is given by

$$
\mathcal{R}_{\mathbf{u}}=\frac{1}{\sqrt{2}}\left(\begin{array}{cc}
1 & -1 \\
1 & 1
\end{array}\right) .
$$

Assume that $\mathcal{R}_{\mathbf{u}}(E[\mathbf{Y}]-E[\mathbf{X}]) \geq \mathbf{0}$, i.e., that $E[\mathbf{Y}]$ belongs to oriented sub-orthant with vertex in $E[\mathbf{X}]$ and oriented by the vector $\mathbf{u}$. Under the assumptions above it is clear that $\operatorname{Var}\left(\mathcal{R}_{\mathbf{u}}^{(r . i)} \mathbf{X}\right)=\operatorname{Var}\left(\mathcal{R}_{\mathbf{u}}^{(r . i)} \mathbf{Y}\right), i=1,2$. Besides,

$$
\operatorname{Cov}\left(\mathcal{R}_{\mathbf{u}}^{(r .1)} \mathbf{X}, \mathcal{R}_{\mathbf{u}}^{(r .2)} \mathbf{X}\right)=\frac{1}{2}\left(\operatorname{Var}\left(X_{1}\right)-\operatorname{Var}\left(X_{2}\right)\right)
$$

and

$$
\operatorname{Cov}\left(\mathcal{R}_{\mathbf{u}}^{(r .1)} \mathbf{Y}, \mathcal{R}_{\mathbf{u}}^{(r .2)} \mathbf{Y}\right)=\frac{1}{2}\left(\operatorname{Var}\left(Y_{1}\right)-\operatorname{Var}\left(Y_{2}\right)\right)
$$


Therefore, from Theorem 3.3.21 in Müller and Stoyan [18] it follows that if

$$
\left(\operatorname{Var}\left(X_{1}\right)-\operatorname{Var}\left(X_{2}\right)\right) \leq\left(\operatorname{Var}\left(Y_{1}\right)-\operatorname{Var}\left(Y_{2}\right)\right),
$$

then $\mathbf{X} \leq_{E_{\mathbf{u}}} \mathbf{Y}$. By using Property 3.4, we get both $X_{1}-X_{2} \leq_{s t} Y_{1}-Y_{2}$ and $X_{1}+X_{2} \leq_{s t} Y_{1}+Y_{2}$.

The following property describes sufficient conditions to compare normal random vectors in the extremality order sense. Other sufficient conditions for the extremality comparison will be stated next.

Property 3.6. Let $\mathbf{X} \sim N\left(\mu_{\mathbf{X}}, \Sigma_{\mathbf{Y}}\right)$ and $\mathbf{Y} \sim N\left(\mu_{\mathbf{Y}}, \Sigma_{\mathbf{Y}}\right)$ be two normally distributed random vectors, and let $\mathbf{u}$ be a unit vector such that $\mathcal{R}_{\mathbf{u}}\left(\mu_{\mathbf{Y}}-\mu_{\mathbf{X}}\right) \geq$ 0 .

a) If $\Sigma_{\mathbf{X}}=\Sigma_{\mathbf{Y}} \Longrightarrow \mathbf{X} \leq_{E_{\mathbf{u}}} \mathbf{Y}$

b) If $\Sigma_{\mathbf{Y}}=\Sigma_{\mathbf{X}}+A$, where $A$ is a matrix such that $\Sigma_{\mathbf{Y}}$ is nonnegative definite and $\mathcal{R}_{\mathbf{u}} A \mathcal{R}_{\mathbf{u}}^{\prime}$ has nonnegative components with zero diagonal elements, then $\mathbf{X} \leq_{E_{\mathbf{u}}} \mathbf{Y}$.

Proof. Part a) follows from fact that $\mu_{\mathcal{R}_{\mathrm{u}} \mathrm{X}} \leq \mu_{\mathcal{R}_{\mathrm{u}} \mathrm{Y}}$ and $\Sigma_{\mathcal{R}_{\mathrm{u}} \mathrm{X}}=\Sigma_{\mathcal{R}_{\mathrm{u}} \mathrm{Y}}$. Therefore $\mathcal{R}_{\mathbf{u}} \mathbf{X} \leq_{s t} \mathcal{R}_{\mathbf{u}} \mathbf{Y}$ by Theorem 3.3.13. in Müller and Stoyan [18], thus $\mathbf{X} \leq_{E_{\mathbf{u}}} \mathbf{Y}$. Part b) follows easily from Theorem 3.3.21.(a) in Müller and Stoyan [18] since $\mu_{\mathcal{R}_{\mathbf{u}} \mathbf{x}} \leq \mu_{\mathcal{R}_{\mathbf{u}} \mathbf{Y}}$ and $\Sigma_{\mathcal{R}_{\mathbf{u}} \mathbf{Y}}=\Sigma_{\mathcal{R}_{\mathbf{u}} \mathbf{x}}+\mathcal{R}_{\mathbf{u}} A \mathcal{R}_{\mathbf{u}}^{\prime}$.

For the next statement, let the oriented upper set in the direction $\mathbf{u}$ (denoted by $U_{\mathbf{u}}$ ) be a set such that $\mathbf{x} \in U_{\mathbf{u}}$ implies $\mathcal{C}_{\mathbf{x}}^{\mathbf{u}} \subset U_{\mathbf{u}}$. 
Property 3.7. Let $\mathbf{X}=\left(X_{1}, \ldots, X_{n}\right)^{\prime}$ and $\mathbf{Y}=\left(Y_{1}, \ldots, Y_{n}\right)^{\prime}$ be two random vectors. If $E\left[I_{U_{\mathbf{u}}}(\mathbf{X})\right] \leq E\left[I_{U_{\mathbf{u}}}(\mathbf{Y})\right]$ for all oriented upper set $U_{\mathbf{u}}$ in the direction $\mathbf{u}$, then $\mathbf{X} \leq_{E_{\mathbf{u}}} \mathbf{Y}$.

Proof. Since the assumption is equivalent to $\mathcal{R}_{\mathbf{u}} \mathbf{X} \leq_{s t} \mathcal{R}_{\mathbf{u}} \mathbf{Y}$, then $\mathcal{R}_{\mathbf{u}} \mathbf{X} \leq_{u o}$ $\mathcal{R}_{\mathbf{u}} \mathbf{Y}$. Thus, $\mathbf{X} \leq_{E_{\mathbf{u}}} \mathbf{Y}$.

Another sufficient condition for the extremality order, easily checked in practice, is stated in the next property. Recall that two random vectors $\mathbf{X}$ and $\mathbf{Y}$ with densities $f_{\mathbf{X}}$ and $f_{\mathbf{Y}}$, respectively, are such that $\mathbf{X}$ is smaller than $\mathbf{Y}$ in the likelihood ratio order (denoted $\mathbf{X} \leq_{l r} \mathbf{Y}$ ) if

$$
f_{\mathbf{X}}(\mathbf{x}) f_{\mathbf{Y}}(\mathbf{y}) \leq f_{\mathbf{X}}(\mathbf{x} \vee \mathbf{y}) f_{\mathbf{Y}}(\mathbf{x} \wedge \mathbf{y})
$$

for all $\mathbf{x}, \mathbf{y} \in \mathbb{R}^{n}$. Since the likelihood ratio order implies the usual stochastic order, the following property immediately follows from the chain of implications

$$
\mathcal{R}_{\mathbf{u}} \mathbf{X} \leq_{l r} \mathcal{R}_{\mathbf{u}} \mathbf{Y} \Rightarrow \mathcal{R}_{\mathbf{u}} \mathbf{X} \leq_{s t} \mathcal{R}_{\mathbf{u}} \mathbf{Y} \Rightarrow \mathcal{R}_{\mathbf{u}} \mathbf{X} \leq_{u o} \mathcal{R}_{\mathbf{u}} \mathbf{Y} \Rightarrow \mathbf{X} \leq_{E_{\mathbf{u}}} \mathbf{Y}
$$

Property 3.8. Let $\mathbf{X}$ and $\mathbf{Y}$ be two random vectors with densities $f_{\mathbf{X}}$ and $f_{\mathbf{Y}}$, respectively. If

$$
f_{\mathbf{X}}\left(\mathcal{R}_{\mathbf{u}}^{\prime} \mathbf{x}\right) f_{\mathbf{Y}}\left(\mathcal{R}_{\mathbf{u}}^{\prime} \mathbf{y}\right) \leq f_{\mathbf{X}}\left(\mathcal{R}_{\mathbf{u}}^{\prime}(\mathbf{x} \vee \mathbf{y})\right) f_{\mathbf{Y}}\left(\mathcal{R}_{\mathbf{u}}^{\prime}(\mathbf{x} \wedge \mathbf{y})\right)
$$

for all $\mathbf{x}, \mathbf{y} \in \mathbb{R}^{n}$, then $\mathbf{X} \leq_{E_{\mathbf{u}}} \mathbf{Y}$.

Proof. Since $\mathcal{R}_{\mathbf{u}}$ is an orthogonal matrix, it follows that the Jacobian of the transformations $\mathcal{R}_{\mathbf{u}} \mathbf{X}$ and $\mathcal{R}_{\mathbf{u}} \mathbf{Y}$ is equal to 1 . Moreover, we also have that 
$\mathcal{R}_{\mathbf{u}}^{-1}=\mathcal{R}_{\mathbf{u}}^{\prime}$. Therefore, $f_{\mathcal{R}_{\mathbf{u}} \mathbf{X}}(\mathbf{x})=f_{\mathbf{X}}\left(\mathcal{R}_{\mathbf{u}}^{\prime} \mathbf{x}\right)$ and $f_{\mathcal{R}_{\mathbf{u}} \mathbf{Y}}(\mathbf{x})=f_{\mathbf{Y}}\left(\mathcal{R}_{\mathbf{u}}^{\prime} \mathbf{x}\right)$ for all $\mathbf{x} \in \mathbb{R}^{n}$. Hence, (3.9) iff $f_{\mathcal{R}_{\mathbf{u}} \mathbf{x}}(\mathbf{x}) f_{\mathcal{R}_{\mathbf{u}} \mathbf{Y}}(\mathbf{y}) \leq f_{\mathcal{R}_{\mathbf{u}} \mathbf{X}}(\mathbf{x} \vee \mathbf{y}) f_{\mathcal{R}_{\mathbf{u}} \mathbf{Y}}(\mathbf{x} \wedge \mathbf{y})$ for all $\mathbf{x}, \mathbf{y} \in \mathbb{R}^{n}$. We thus get $\mathcal{R}_{\mathbf{u}} \mathbf{X} \leq_{l r} \mathcal{R}_{\mathbf{u}} \mathbf{Y}$ and consequently $\mathbf{X} \leq_{E_{\mathbf{u}}} \mathbf{Y}$.

A random vector $\mathbf{X}$ has the $M T P_{2}$ property if $\mathbf{X} \leq_{l r} \mathbf{X}$ (see for instance Karlin and Rinott [8]). For $\mathbf{X}$ normally distributed it has a $M T P_{2}$ iff the off-diagonal elements of $\Sigma_{\mathbf{X}}^{-1}$ are nonpositive. Particulary a bivariate normal density is $M T P_{2}$ if the correlation coefficient is nonnegative (Karlin and Rinott [9]). We show in Example 3.9 that for any normally distributed bivariate random vector $\mathbf{X}$, always there exists a rotation $\mathcal{R}_{\mathbf{u}}$ such that $\mathcal{R}_{\mathbf{u}} \mathbf{X}$ has the $M T P_{2}$ property. In fact, the same example also provides a sufficient condition for extremality order in terms of the likelihood order.

Example 3.9. Consider $\mathbf{X}=\left(X_{1}, X_{2}\right)^{\prime}$ a normally distributed random vector with covariance matrix $\Sigma_{\mathbf{X}}$. It is easily seen that $\Sigma_{\mathbf{X}}=Q D Q^{\prime}$ where $Q=\left(q_{i j}\right)$ is an orthogonal matrix and $D=\left(d_{i i}\right)$ is a diagonal matrix with nonnegative elements and $d_{11} \geq d_{22}$. Let $\mathbf{u}=\left(q_{11}, q_{21}\right)^{\prime}$ be the first column of the matrix $Q$. Then, according to (3.1),

$$
\mathcal{R}_{\mathbf{u}}=\frac{\sqrt{2}}{2}\left(\begin{array}{ll}
q_{11}+q_{21} & q_{21}-q_{11} \\
q_{11}-q_{21} & q_{11}+q_{21}
\end{array}\right)
$$

The vector $\mathcal{R}_{\mathbf{u}} \mathbf{X}$ also is normaly distributed and $\boldsymbol{\Sigma}_{\mathcal{R}_{\mathbf{u}} \mathbf{X}}=\mathcal{R}_{\mathbf{u}} \boldsymbol{\Sigma}_{\mathbf{X}} \mathcal{R}_{\mathbf{u}}^{\prime}$ (see, e.g., Valdez and Dhaene [25], Theorem 2).

It is clear that

$$
\boldsymbol{\Sigma}_{\mathcal{R}_{\mathbf{u}} \mathbf{X}}=\mathcal{R}_{\mathbf{u}} Q D Q^{\prime} \mathcal{R}_{\mathbf{u}}^{\prime}=\frac{1}{2}\left(\begin{array}{cc}
d_{11}+d_{22} & d_{11}-d_{22} \\
d_{11}-d_{22} & d_{11}+d_{22}
\end{array}\right)
$$


As $d_{11} \geq d_{22}$ we have that $\mathcal{R}_{\mathbf{u}} \mathbf{X}$ has the $M T P_{2}$ property. If we take $\mathbf{Y}=\mathbf{X}+\mathcal{R}_{\mathbf{u}}^{\prime} \mathbf{s}$ where $\mathbf{s}=\left(s_{1}, s_{2}\right)^{\prime}$ is such that

$$
\frac{d_{11}-d_{22}}{d_{11}+d_{22}} \leq \frac{s_{2}}{s_{1}} \leq \frac{d_{11}+d_{22}}{d_{11}-d_{22}},
$$

then from Theorem 3.2-b in Rinott and Scarsini [21] we conclude that $\mathcal{R}_{\mathbf{u}} \mathbf{X} \leq_{l r}$ $\mathcal{R}_{\mathbf{u}}\left(\mathbf{X}+\mathcal{R}_{\mathbf{u}}^{\prime} \mathbf{s}\right)$

and so, $\mathbf{X} \leq_{E_{\mathbf{u}}} \mathbf{Y}$.

The extremality order satisfies the following closure properties (closure with respect to convergence in distribution and closure with respect to mixture). Here, the notation $[Z \mid A]$ stands for the random object whose distribution is the conditional distribution of $Z$ given the event $A$.

Property 3.10. a) Let $\left\{\mathbf{X}_{j}, j=1,2, \ldots\right\}$ and $\left\{\mathbf{X}_{j}, j=1,2, \ldots\right\}$ be two sequences of random vectors such that $\mathbf{X}_{j} \longrightarrow_{d} \mathbf{X}$ and $\mathbf{Y}_{j} \longrightarrow_{d} \mathbf{Y}$ as $j \longrightarrow \infty$, where $\longrightarrow_{d}$ denotes convergence in distribution. If $\mathbf{X}_{j} \leq_{E_{\mathbf{u}}}$ $\mathbf{Y}_{j}$ for all $j=1,2, \ldots$, then $\mathbf{X} \leq_{E_{\mathbf{u}}} \mathbf{Y}$.

b) Let $\mathbf{X}, \mathbf{Y}$ and $\Theta$ be random vectors such that $[\mathbf{X} \mid \Theta=\theta] \leq_{E_{\mathbf{u}}}[\mathbf{Y} \mid \Theta=\theta]$ for all $\theta$ in the support of $\Theta$. Then $\mathbf{X} \leq_{E_{\mathbf{u}}} \mathbf{Y}$.

Proof. a) Clearly, if $\mathbf{X}_{j} \longrightarrow_{d} \mathbf{X}$ and $\mathbf{Y}_{j} \longrightarrow_{d} \mathbf{Y}$ as $j \longrightarrow \infty$, then $\mathcal{R}_{\mathbf{u}} \mathbf{X}_{j} \longrightarrow_{d}$ $\mathcal{R}_{\mathbf{u}} \mathbf{X}$ and $\mathcal{R}_{\mathbf{u}} \mathbf{Y}_{j} \longrightarrow_{d} \mathcal{R}_{\mathbf{u}} \mathbf{Y}$ as $j \longrightarrow \infty$. But $\mathcal{R}_{\mathbf{u}} \mathbf{X}_{j} \leq_{u o} \mathcal{R}_{\mathbf{u}} \mathbf{Y}_{j}$ since $\mathbf{X}_{j} \leq_{E_{\mathbf{u}}} \mathbf{Y}_{j}$, for $j=1,2, \ldots$. Applying Theorem 6.G.3-d in [24], it follows that $\mathcal{R}_{\mathbf{u}} \mathbf{X} \leq_{u o} \mathcal{R}_{\mathbf{u}} \mathbf{Y}$; thus also $\mathbf{X} \leq_{E_{\mathbf{u}}} \mathbf{Y}$. b) From $[\mathbf{X} \mid \Theta=\theta] \leq_{E_{\mathbf{u}}}[\mathbf{Y} \mid \Theta=\theta]$, it follows that $\left[\mathcal{R}_{\mathbf{u}} \mathbf{X} \mid \Theta=\theta\right] \leq_{u o}\left[\mathcal{R}_{\mathbf{u}} \mathbf{Y} \mid \Theta=\theta\right]$. The assertion follows from Theorem 6.G.3-e in Shaked and Shanthikumar [24]. 
The following statement describes a property that will be used in Section 4. Recall that the copula $\mathrm{C}$ of a random vector $\mathbf{X}$ is a cumulative distribution function with uniform marginals on $[0,1]$ such that, for all $\mathbf{x} \in \mathbb{R}^{n}$,

$$
F_{\mathbf{X}}\left(x_{1}, \ldots, x_{n}\right)=C\left(F_{X_{1}}\left(x_{1}\right), \ldots, F_{X_{n}}\left(x_{n}\right)\right)
$$

The copula of the vector $\mathbf{X}$ describes dependence properties of its components, and it is unique if $F_{X_{1}}, \ldots, F_{X_{n}}$ are continuous. For more details about copulas, see Nelsen [19].

Theorem 3.11. Let $\mathbf{X}=\left(X_{1}, \ldots, X_{n}\right)^{\prime}$ and $\mathbf{Y}=\left(Y_{1}, \ldots, Y_{n}\right)^{\prime}$ be two random vectors and $\mathbf{u}=\left(u_{1}, \ldots, u_{n}\right)$ a unit vector. If $\mathcal{R}_{\mathbf{u}} \mathbf{X}$ and $\mathcal{R}_{\mathbf{u}} \mathbf{Y}$ have the same copula, then

$$
\mathbf{X} \leq_{E_{\mathbf{u}}} \mathbf{Y} \Longrightarrow \phi\left(\mathcal{R}_{\mathbf{u}}^{(1)} \mathbf{X}, \ldots, \mathcal{R}_{\mathbf{u}}^{(n)} \mathbf{X}\right) \leq_{s t} \phi\left(\mathcal{R}_{\mathbf{u}}^{(1)} \mathbf{Y}, \ldots, \mathcal{R}_{\mathbf{u}}^{(n)} \mathbf{Y}\right)
$$

for every increasing function $\phi$. In particular, denoted with $\mathcal{R}_{\mathbf{u}}^{(c . i)}$ the $i$-th column of the matrix $\mathcal{R}_{\mathbf{u}}$, and the assumptions it holds

$$
\mathbf{X} \leq_{E_{\mathbf{u}}} \mathbf{Y} \Longrightarrow \sum_{i=1}^{n} \mathbf{a}^{\prime} \mathcal{R}_{\mathbf{u}}^{(c . i)} X_{i} \leq_{s t} \sum_{i=1}^{n} \mathbf{a}^{\prime} \mathcal{R}_{\mathbf{u}}^{(c . i)} Y_{i}
$$

for every vector $\mathbf{a}=\left(a_{1}, \ldots, a_{n}\right)^{\prime}$ with nonnegative components.

Proof. As $\mathbf{X} \leq_{E_{\mathbf{u}}} \mathbf{Y}$, then from Property 3.4, $\mathcal{R}_{\mathbf{u}}^{(r . i)} \mathbf{X} \leq_{s t} \mathcal{R}_{\mathbf{u}}^{(r . i)} \mathbf{Y}$ for all $i=1, \ldots, n$. Since $\mathcal{R}_{\mathbf{u}} \mathbf{X}$ and $\mathcal{R}_{\mathbf{u}} \mathbf{Y}$ have the same copula, by Theorem 6.B.14. in Shaked and Shanthikumar [24] it follows that $\mathcal{R}_{\mathbf{u}} \mathbf{X} \leq_{s t} \mathcal{R}_{\mathbf{u}} \mathbf{Y}$. Now, by Theorem 3.3.11 in Müller and Stoyan [18], the first assertion follows. In particular, letting $\phi\left(x_{1}, \ldots, x_{n}\right)=\left(a_{1}, \ldots, a_{n}\right)\left(x_{1}, \ldots, x_{n}\right)^{\prime}$, where $\mathbf{a}=$ $\left(a_{1}, \ldots, a_{n}\right)^{\prime}$ is a vector with non-negative components, we get

$$
\mathbf{a}^{\prime} \mathcal{R}_{\mathbf{u}} \mathbf{X} \leq_{s t} \mathbf{a}^{\prime} \mathcal{R}_{\mathbf{u}} \mathbf{Y}
$$


i.e., the second assertion.

Note that, in particular, Theorem 3.11 applies when the unit vector $\mathbf{u}$ is such that $\mathcal{R}_{\mathbf{u}} \mathbf{X}$ and $\mathcal{R}_{\mathbf{u}} \mathbf{Y}$ have the copula $C(u, v)=u v$, i.e., when they have independent components. To find a unit vector $\mathbf{u}$ such that $\mathcal{R}_{\mathbf{u}} \mathbf{X}$ and $\mathcal{R}_{\mathbf{u}} \mathbf{Y}$ have the same copula in some situations it is easy. For example, assume that $\mathbf{X}$ and $\mathbf{Y}$ are normally distributed with covariance matrix $\Sigma_{\mathbf{X}}$ and $\Sigma_{\mathbf{Y}}$, respectively. Assume that the eigenvectors $\overrightarrow{v_{1}}, \overrightarrow{v_{2}}$ of $\Sigma_{\mathbf{X}}$ and $\Sigma_{\mathbf{Y}}$ are the same. If we define $\vec{p}=\frac{\overrightarrow{v_{1}}}{\|\overrightarrow{v \vec{v}}\|}+\frac{\overrightarrow{v_{2}}}{\left\|\overrightarrow{v_{2}}\right\|}$ and $\mathbf{u}=\frac{\vec{p}}{\|\vec{p}\|}$, then $\mathcal{R}_{\mathbf{u}} \mathbf{X}$ and $\mathcal{R}_{\mathbf{u}} \mathbf{Y}$ will have the same copula. A graphical representation of this situation is shown in Figure 1

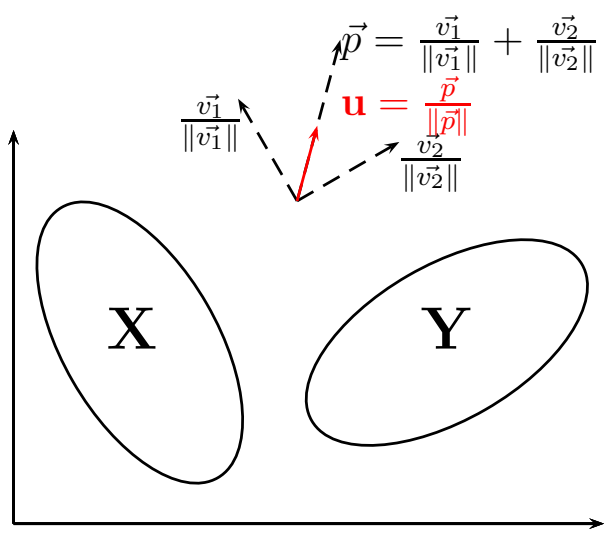

(a)

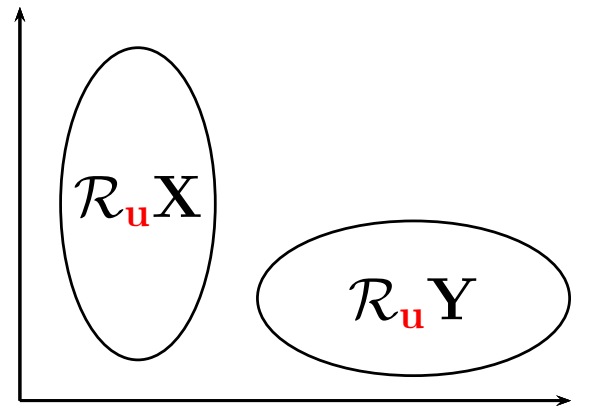

(b)

Figure 1: Rotations of bivariate normal distributions: (a) different copula; (b) same copula.

Figure 1 shows that in the original system, before the rotation, $\mathbf{X}$ has a negative dependency and $\mathbf{Y}$ has a positive dependence. Therefore, their copulas are different. After of the rotation in the directions $\mathbf{u}$, indicated above, $\mathcal{R}_{\mathbf{u}} \mathbf{X}$ and $\mathcal{R}_{\mathbf{u}} \mathbf{Y}$ have the same copula, i.e., $C(u, v)=u v$. It is inter- 
esting to observe that, if $\mathbf{X}$ and $\mathbf{Y}$ have a common copula, $\mathcal{R}_{\mathbf{u}} \mathbf{X}$ and $\mathcal{R}_{\mathbf{u}} \mathbf{Y}$ may not have common copula. In fact, consider $\mathbf{X}$ to have $N\left(\mu, I_{2}\right)$ distribution and $\mathbf{Y}$ have $N\left(\mu, D_{2}\right)$ distribution, where $I_{2}$ is the identical matrix and $D_{2}$ is a diagonal matrix with $d_{11}>d_{22}$. Clearly, $\mathbf{X}$ has a spherical distribution, and $\mathbf{X}$ and $\mathbf{Y}$ have a common copula. Let now $\mathbf{u}=[1,0]^{\prime}$ be the rotation vector. In the Figure 2 is shown that $\mathcal{R}_{\mathbf{u}} \mathbf{X}$ has the same copula as before the rotation, but $\mathcal{R}_{\mathbf{u}} \mathbf{Y}$ has a different copula copula since positive dependency can be observed.

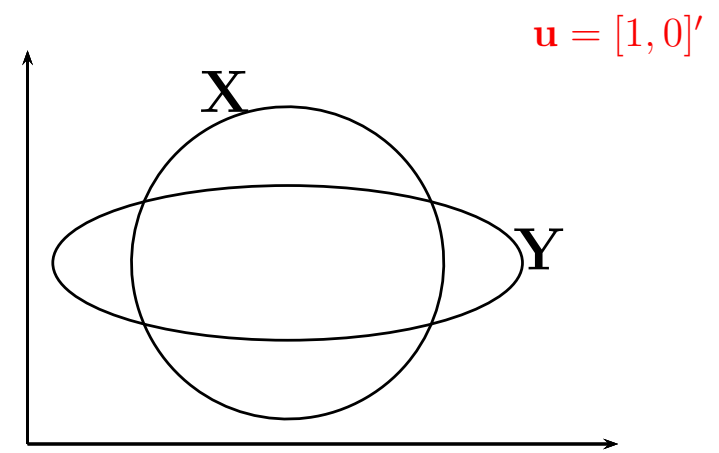

(a)

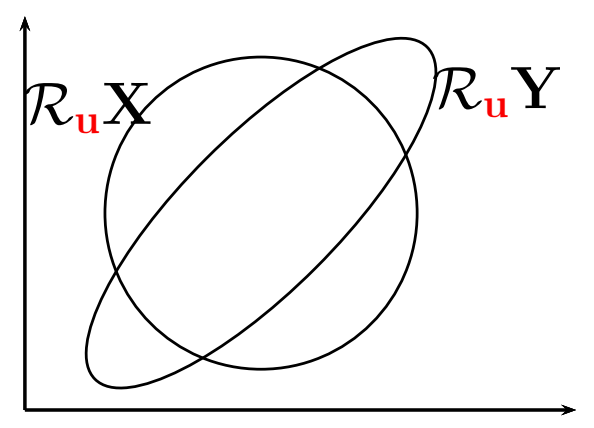

(b)

Figure 2: Rotations of bivariate normal distributions: (a) same copula; (b) different copula

Corollary 3.12. Under the same assuptions of Theorem 3.11 it holds that

$$
\mathbf{X} \leq_{E_{\mathbf{u}}} \mathbf{Y} \Longrightarrow \mathbf{u}^{\prime} \mathbf{X} \leq_{s t} \mathbf{u}^{\prime} \mathbf{Y}
$$

Proof. We need only consider $\mathbf{a}=[1, \ldots, 1]^{\prime}$ and the assertion follows immediately from Theorem 3.11 and formula (3.7) 
The Corollary 3.12 follows also from the fact that if two random vectors are ordered in the multivariate stochastic order, then the sum of their components are ordered in the univariate stochastic order (see, e.g. Theorem 6.B.16-a in Shaked and Shanthikumar [24]). In Theorem 3.11 and Corollary 3.12 it is worth noting that the coefficients $\mathbf{a}^{\prime} \mathcal{R}_{\mathbf{u}}^{(c . i)}$ and components of $\mathbf{u}^{\prime}$ may also be negative; thus Theorem 3.11 gives conditions to compare, in usual stochastic order, non-positive linear combinations of dependent random variables. If the vectors $\mathbf{X}$ and $\mathbf{Y}$ have the same means, then the following property holds.

Theorem 3.13. Let $\mathbf{X}=\left(X_{1}, \ldots, X_{n}\right)^{\prime}$ and $\mathbf{Y}=\left(Y_{1}, \ldots, Y_{n}\right)^{\prime}$ be two random vectors such that $E[\mathbf{X}]=E[\mathbf{Y}]$. If there exists a vector $\mathbf{u}$ such that $\mathbf{X} \leq_{E_{\mathbf{u}}} \mathbf{Y}$, then

$$
\mathcal{R}_{\mathbf{u}}^{(r . i)} \mathbf{X}={ }_{s t} \mathcal{R}_{\mathbf{u}}^{(r . i)} \mathbf{Y}, \text { for all } i=1, \ldots, n
$$

Proof. Since $\mathbf{X} \leq_{E_{\mathbf{u}}} \mathbf{Y}$, from Property 3.4, we have $\mathcal{R}_{\mathbf{u}}^{(r . i)} \mathbf{X} \leq_{s t} \mathcal{R}_{\mathbf{u}}^{(r . i)} \mathbf{Y}, \quad i=$ $1, \ldots, n$. Observe that

$$
E[\mathbf{X}]=E[\mathbf{Y}] \Longrightarrow \mathcal{R}_{\mathbf{u}} E[\mathbf{X}]=\mathcal{R}_{\mathbf{u}} E[\mathbf{Y}] \Longrightarrow E\left[\mathcal{R}_{\mathbf{u}} \mathbf{X}\right]=E\left[\mathcal{R}_{\mathbf{u}} \mathbf{Y}\right]
$$

Now the proof follows from the fact that variables ordered in usual stochastic order having the same mean should also have the same distribution (see Theorem 1.A.8. in Shaked and Shanthikumar [24]).

Relationships between extremality order and two positive dependence orders are obtained in the next result.

Theorem 3.14. Let $\mathbf{X}=\left(X_{1}, \ldots, X_{n}\right)^{\prime}$ and $\mathbf{Y}=\left(Y_{1}, \ldots, Y_{n}\right)^{\prime}$ be two random vectors such that $E[\mathbf{X}]=E[\mathbf{Y}]$. If $\mathbf{X} \leq_{E_{\mathbf{u}}} \mathbf{Y}$ then $\mathcal{R}_{\mathbf{u}} \mathbf{X} \leq_{P Q D} \mathcal{R}_{\mathbf{u}} \mathbf{Y}$. Moreover, if $n=2$, it follows also that $\mathcal{R}_{\mathbf{u}} \mathbf{X} \leq_{\text {sm }} \mathcal{R}_{\mathbf{u}} \mathbf{Y}$. 
Proof. From Theorem 3.13 it is easily seen that $\mathcal{R}_{\mathbf{u}} \mathbf{X}$ and $\mathcal{R}_{\mathbf{u}} \mathbf{Y}$ have the same marginals. Since $\mathbf{X} \leq_{E_{\mathbf{u}}} \mathbf{Y} \Longleftrightarrow \mathcal{R}_{\mathbf{u}} \mathbf{X} \leq_{u o} \mathcal{R}_{\mathbf{u}} \mathbf{Y}$, the first assertion follows from definition of positive quadrant dependence order, while the second from the equivalency between $\leq_{P D Q}$ and $\leq_{s m}$ when $n=2$.

Corollary 3.15. Let $\mathbf{X}=\left(X_{1}, X_{2}\right)^{\prime}$ and $\mathbf{Y}=\left(Y_{1}, Y_{2}\right)^{\prime}$ be two random vectors such that $E[\mathbf{X}]=E[\mathbf{Y}]$. If $\mathbf{X} \leq_{E_{\mathbf{u}}} \mathbf{Y}$ then $\mathbf{a}^{\prime} \mathcal{R}_{\mathbf{u}} \mathbf{Y} \leq_{c v} \mathbf{a}^{\prime} \mathcal{R}_{\mathbf{u}} \mathbf{X}$ for all $\mathbf{a} \in \mathbf{R}^{2}$ such that $a_{1} a_{2} \geq 0$.

Proof. According to Theorem 3.14, it holds $\mathcal{R}_{\mathbf{u}} \mathbf{X} \leq_{\text {sm }} \mathcal{R}_{\mathbf{u}} \mathbf{Y}$. Hence, from Theorem 9.A.9 in Shaked and Shanthikumar [24],

$$
\left(\begin{array}{cc}
a_{1} & 0 \\
0 & a_{2}
\end{array}\right) \mathcal{R}_{\mathbf{u}} \mathbf{X} \leq_{s m}\left(\begin{array}{cc}
a_{1} & 0 \\
0 & a_{2}
\end{array}\right) \mathcal{R}_{\mathbf{u}} \mathbf{Y}
$$

for all $a_{1}, a_{2}$ such that $a_{1} a_{2} \geq 0$. Combining (3.11) and (9.A.19) in Shaked in Shanthikumar [24], we can assert that $\mathbf{a}^{\prime} \mathcal{R}_{\mathbf{u}} \mathbf{X} \leq_{c x} \mathbf{a}^{\prime} \mathcal{R}_{\mathbf{u}} \mathbf{Y}$ and $\mathbf{a}^{\prime} \mathcal{R}_{\mathbf{u}} \mathbf{Y} \leq_{c v}$ $\mathbf{a}^{\prime} \mathcal{R}_{\mathbf{u}} \mathrm{X}$

As a particular case of Corollary 3.15, with $\mathbf{a}=\frac{1}{\sqrt{2}}[1,1]^{\prime}$, we can also conclude that, under the same assumptions,

$$
\mathbf{X} \leq_{E_{\mathbf{u}}} \mathbf{Y} \Longrightarrow \mathbf{u}^{\prime} \mathbf{Y} \leq_{c v} \mathbf{u}^{\prime} \mathbf{X}
$$

We finish this section with a necessary condition for the extremality order. To this end, recall that given two non-negative random variables $X$ and $Y$, $X$ is said to be smaller than $Y$ in Laplace transform order (briefly $X \leq_{L t} Y$ ) if only if $E\left[\exp ^{-s X}\right] \geq E\left[\exp ^{-s Y}\right]$, for all $s \in \mathbb{R}^{+}$. 
Theorem 3.16. Let $\mathbf{X}, \mathbf{Y}$ be two random vectors and $\mathbf{u}$ a unit vector such that $\mathcal{R}_{\mathbf{u}} \mathbf{X}$ and $\mathcal{R}_{\mathbf{u}} \mathbf{Y}$ are positive. If $\mathbf{X} \leq_{E_{(-\mathbf{u})}} \mathbf{Y}$ then $\mathbf{u}^{\prime} \mathbf{X} \geq_{L t} \mathbf{u}^{\prime} \mathbf{Y}$ and, in particular, $E\left[\mathbf{u}^{\prime} \mathbf{X}\right] \geq E\left[\mathbf{u}^{\prime} \mathbf{Y}\right]$.

Proof. Since $\mathbf{X} \leq_{E_{(-\mathbf{u})}} \mathbf{Y} \Longleftrightarrow \mathcal{R}_{\mathbf{u}} \mathbf{X} \geq_{l o} \mathcal{R}_{\mathbf{u}} \mathbf{Y}$, and since $\mathcal{R}_{\mathbf{u}} \mathbf{X}$ and $\mathcal{R}_{\mathbf{u}} \mathbf{Y}$ are positive, then from Theorem 6.G.14 in Shaked and Shanthikumar [24] it follows

$$
\sum_{i=1}^{n} a_{i} \mathcal{R}_{\mathbf{u}}^{(r . i)} \mathbf{X} \geq_{L t} \sum_{i=1}^{n} a_{i} \mathcal{R}_{\mathbf{u}}^{(r . i)} \mathbf{Y}, \quad \text { whenever } a_{i} \geq 0, i=1,2, \ldots, n .
$$

Assuming $a_{i}=\frac{1}{\sqrt{n}}$, for all $i=1,2, \ldots, n$, the assertion follows from $\mathbf{1}^{\prime} \mathcal{R}_{\mathbf{u}} \mathbf{X}=$ $\sqrt{n} \mathbf{u}^{\prime} \mathbf{X}$. The second inequality follows as a consequence of the comparison in Laplace transform order.

\section{Portfolio comparisons with extremality order}

In this section we describe some examples of application of previous results to the the single period portfolio problem. We first describe the problem. Consider an economic agent with unitary initial capital. Suppose that the random variables $X_{1}, \cdots, X_{n}$ represent the outcome of $n$ financial positions which can be chosen for investment. Thus we have risks $-X_{1}, \cdots,-X_{n}$. In this context, a portfolio is a random variable $Z_{\mathbf{a}}=\sum_{i=1}^{n} a_{i} X_{i}$, where the weights vector $\mathbf{a}=\left(a_{1}, \ldots, a_{n}\right)$ range in the subset $\mathbf{A}_{n}=\left\{\mathbf{a}=\left(a_{1}, \ldots, a_{n}\right)\right.$ : $\left.\sum_{i=1}^{n} a_{i}=1, a_{i} \geq 0, i=1, \ldots, n\right\}$. When short selling are permitted, then the condition $a_{i} \geq 0$ can be removed. The goal of the single period portfolio problem consists in determining the allocation $\mathbf{a}=\left\{a_{1}, \ldots, a_{n}\right\}$ of the unitary wealth to the $n$ risks that minimize the total risk, or that maximize the expected utility of the resulting final wealth $Z_{\mathbf{a}}$. 
A first problem that the economic agent can consider is the minimization of the risk, which is commonly expressed as the minimization of the value at risk at a fixed quantile $\alpha\left(V a R_{\alpha}\right)$ of the random loss $-Z_{\mathbf{a}}$ (see, e.g., Jorion [7]). It represents the $\alpha$-quantile of the loss distribution of portfolio. This means that $V a R_{\alpha}$ is the better loss among the $(1-\alpha) 100 \%$ worst losses, and it is formally defined as follows: if $F$ is the distribution of $-Z_{\mathbf{a}}$ and $\alpha \in(0,1)$, then

$$
\operatorname{VaR}_{\alpha}(X)=\inf \{x \in \mathbb{R} ; \mid F(x) \geq \alpha\}
$$

Let us first consider the case that the risk manager wants to allocate the wealth to $n$ risks, and he/she has to chose between two sets of dependent financial positions, say $\mathbf{X}=\left(X_{1}, \ldots, X_{n}\right)^{\prime}$ or $\mathbf{Y}=\left(Y_{1}, \ldots, Y_{n}\right)^{\prime}$. Assume that there exists a vector $\mathbf{u}=\left(u_{1}, \ldots, u_{n}\right)^{\prime}$ such that the assumptions of Theorem 3.11 are satisfied (i.e., $\mathbf{X} \leq_{E_{\mathbf{u}}} \mathbf{Y}$ and the rotated vectors have the same copula). Let $\mathbf{a}=\left(a_{1}, \ldots, a_{n}\right)^{\prime}$ be any vector such that $\frac{\mathbf{a}}{\mathbf{a}^{\prime} \mathcal{R}_{\mathbf{u}} \mathbf{1}} \geq \mathbf{0}$. Since the usual stochastic order implies the corresponding order between values at risk for every $\alpha$ (see, e.g., Bäuerle and Müller [2]), then, for every allocation

$$
\omega=\left(\frac{\mathbf{a}^{\prime} \mathcal{R}_{\mathbf{u}}^{(c .1)}}{\mathbf{a}^{\prime} \mathcal{R}_{\mathbf{u}} \mathbf{1}}, \ldots, \frac{\mathbf{a}^{\prime} \mathcal{R}_{\mathbf{u}}^{(c . n)}}{\mathbf{a}^{\prime} \mathcal{R}_{\mathbf{u}} \mathbf{1}}\right)^{\prime}
$$

the economic agent will choose the portfolio $Z_{\mathbf{Y}}=\sum_{i=1}^{n} \omega_{i} Y_{i}$ rather than $Z_{\mathbf{X}}=\sum_{i=1}^{n} \omega_{i} X_{i}$. It is interesting to observe that some of the weights $\omega_{i}$ can be negative, so that even portfolios with short selling can be compared.

Similarly, if the economic agent is non-satiable, which means that he/she has an increasing utility function $U$, under the same conditions as above her/his expected utility will be maximized choosing the portfolio $Z_{\mathbf{Y}}$ instead 
of $Z_{\mathbf{X}}$, since $Z_{\mathbf{X}} \leq s t Z_{\mathbf{Y}} \Longrightarrow E\left[U\left(Z_{\mathbf{X}}\right)\right] \leq E\left[U\left(Z_{\mathbf{Y}}\right)\right]$ for any increasing function $U$. To illustrate this result, we introduce an example in the bivariate case.

Example 4.1. Let $\mathbf{X}=\left(X_{1}, X_{2}\right)^{\prime}$ and $\mathbf{Y}=\left(Y_{1}, Y_{2}\right)^{\prime}$ be bivariate normally distributed random vectors with mean $\mu_{\mathbf{X}}$ and $\mu_{\mathbf{Y}}$, respectively and the same covariance matrix $\Sigma$. Choose any unit vector $\mathbf{u}=\left(u_{1}, u_{2}\right)^{\prime}$ such that $\mu_{\mathbf{Y}} \in$ $\mathcal{C}_{\mu_{\mathbf{X}}}^{\mathbf{u}}$, (for example the vector $\left.\mathbf{u}=\frac{\mu_{\mathbf{Y}}-\mu_{\mathbf{X}}}{\left\|\mu_{\mathbf{Y}}-\mu_{\mathbf{X}}\right\|}\right)$. According to (3.1), the rotation matrix is given by

$$
\mathcal{R}_{\mathbf{u}}=\frac{\sqrt{2}}{2}\left(\begin{array}{cc}
u_{1}+u_{2} & u_{2}-u_{1} \\
u_{1}-u_{2} & u_{1}+u_{2}
\end{array}\right) .
$$

It is easy to check that $\mathcal{R}_{\mathbf{u}} \mathbf{X}$ and $\mathcal{R}_{\mathbf{u}} \mathbf{Y}$ have the same copula. Let now $Z_{\mathbf{X}}$ and $Z_{\mathbf{Y}}$ be two portfolios defined as

$$
\begin{aligned}
& Z_{\mathbf{X}}=\frac{1}{2}\left(1+\frac{a_{1} u_{1}-a_{2} u_{2}}{a_{1} u_{2}+a_{2} u_{1}}\right) X_{1}+\frac{1}{2}\left(1-\frac{a_{1} u_{1}-a_{2} u_{2}}{a_{1} u_{2}+a_{2} u_{1}}\right) X_{2} \\
& Z_{\mathbf{Y}}=\frac{1}{2}\left(1+\frac{a_{1} u_{1}-a_{2} u_{2}}{a_{1} u_{2}+a_{2} u_{1}}\right) Y_{1}+\frac{1}{2}\left(1-\frac{a_{1} u_{1}-a_{2} u_{2}}{a_{1} u_{2}+a_{2} u_{1}}\right) Y_{2},
\end{aligned}
$$

where $\mathbf{X}$ and $\mathbf{Y}$ are financial positions which can be chosen for investment. By Theorem 3.11, we have that

$$
Z_{\mathbf{X}} \leq_{s t} Z_{\mathbf{Y}}
$$

for every $\mathbf{a}=\left(a_{1}, a_{2}\right)^{\prime}$ such that $\frac{\mathbf{a}}{\mathbf{a}^{\prime} \mathcal{R}_{\mathbf{u}} \mathbf{1}} \geq \mathbf{0}$. Therefore, if an investor measures the risk through value at risk, then he/she prefers the portfolio $Z_{\mathbf{Y}}$ instead $Z_{\mathbf{Y}}$ since from (4.2) it follows that, $\operatorname{VaR}_{\alpha}\left(-Z_{\mathbf{Y}}\right) \leq \operatorname{VaR}_{\alpha}\left(-Z_{\mathbf{X}}\right)$, for all $\alpha \in(0,1)$. Indeed, if the investor is non-satiable the same conclusion can be drawn. It is also remarkable that the weights of the two portfolios can assume negative values. 
Now, let us consider the case where the economic agent has to chose between two sets of risks, say $\mathbf{X}=\left(X_{1}, X_{2}\right)$ or $\mathbf{Y}=\left(Y_{1}, Y_{2}\right)$, but assume here that $E[\mathbf{X}]=E[\mathbf{Y}]$. Thus the expectation is the same for any linear combination of the risks. In fact, in such a case, given any two portfolios $Z_{\mathbf{X}}=\sum_{i=1}^{2} b_{i} X_{i}$ and $Z_{\mathbf{Y}}=\sum_{i=1}^{2} b_{i} Y_{i}$, they cannot be ordered in usual stochastic order, since they have the same expectations. However, the economic agent can prefer one of the two portfolios if she/he, beside of being non-satiable, is risk averse. In fact, in this situation, the utility function $U$ is increasing and concave (see, e.g., Yaari [26]), and a comparison in the concave order can be used as a criteria to choose between portfolios. By a direct application of Corollary 3.15, it can be asserted that, if $\mathbf{X} \leq_{E_{\mathbf{u}}} \mathbf{Y}$, expected utility of the agent will be greater choosing the portfolio $Z_{\mathbf{X}}=\sum_{i=1}^{2} \mathbf{a}^{\prime} \mathcal{R}_{\mathbf{u}}^{(c . i)} X_{i}$ rather than $Z_{\mathbf{Y}}=\sum_{i=1}^{2} \mathbf{a}^{\prime} \mathcal{R}_{\mathbf{u}}^{(c .)} Y_{i}$, for all $\mathbf{a}=\left[a_{1}, a_{2}\right]^{\prime}$ such that $a_{1} a_{2} \geq 0$. As above, this fact becomes particularly interesting whenever some of the allocations to the risks are negative, thus allowing comparisons also in the case of short selling.

Example 4.2. Let $\mathbf{X}=\left(X_{1}, X_{2}\right)^{\prime}$ and $\mathbf{Y}=\left(Y_{1}, Y_{2}\right)^{\prime}$ be two random vectors elliptically distributed such that $E[\mathbf{X}]=E[\mathbf{Y}]$. Assume that the covariance matrices $\Sigma_{\mathbf{X}}$ and $\Sigma_{\mathbf{Y}}$ have the same eigenvalues $d_{1}$ and $d_{2}$. Let

$$
Q=\left(\begin{array}{cc}
q_{1} & q_{2} \\
q_{2} & -q_{1}
\end{array}\right) \quad \text { and } T=\left(\begin{array}{cc}
t_{1} & t_{2} \\
t_{2} & -t_{1}
\end{array}\right)
$$

be the eigenvectors matrices of $\Sigma_{\mathbf{X}}$ and $\Sigma_{\mathbf{Y}}$, respectively. It is clear that $\Sigma_{\mathbf{X}}=Q D Q^{\prime}$ and $\Sigma_{\mathbf{Y}}=T D T^{\prime}$ where $D=\operatorname{diag}\left(d_{1}, d_{2}\right)$. Let $\mathbf{u}=\frac{(Q+T) \mathbf{1}}{\|(Q+T) \mathbf{1}\|}$ be 
the unit vector, this gives

$$
\mathbf{u}=\frac{1}{\|(Q+T) \mathbf{1}\|}\left[q_{1}+t_{1}+q_{2}+t_{2}, q_{2}+t_{2}-q_{1}-t_{1}\right]^{\prime} .
$$

From (3.1) we have that

$$
\mathcal{R}_{\mathbf{u}}=\frac{\sqrt{2}}{\|(Q+T) \mathbf{1}\|}\left(\begin{array}{cc}
q_{2}+t_{2} & -q_{1}-t_{1} \\
q_{1}+t_{1} & q_{2}+t_{2}
\end{array}\right) .
$$

By straightforward calculations we can see that

$$
\Sigma_{\mathcal{R}_{\mathbf{u}} \mathbf{X}}=\mathcal{R}_{\mathbf{u}} Q D Q^{\prime} \mathcal{R}_{\mathbf{u}}^{\prime} \quad \text { and } \Sigma_{\mathcal{R}_{\mathbf{u}} \mathbf{Y}}=\mathcal{R}_{\mathbf{u}} T D T^{\prime} \mathcal{R}_{\mathbf{u}}^{\prime}
$$

have the same diagonal and their off-diagonal elements are given by $\rho=$ $\left(d_{1}-d_{2}\right)\left(q_{1} t_{1}+q_{2} t_{2}+1\right)\left(q_{1} t_{1}-q_{2} t_{2}\right)$ and $(-\rho)$ respectively. Without lack of generality assume $\rho \leq 0$, then $\mathcal{R}_{\mathbf{u}} \mathbf{X} \leq_{p q d} \mathcal{R}_{\mathbf{u}} \mathbf{Y}$ (see Landsman and Tsanakas [12], Corollary 2.) and consequently $\mathbf{X} \leq_{E_{\mathbf{u}}} \mathbf{Y}$. Therefore, if we consider the random variables

$$
\begin{aligned}
& Z_{\mathbf{X}}=\frac{1}{2}\left(1+\frac{q_{1}+t_{1}}{q_{2}+t_{2}}\right) X_{1}+\frac{1}{2}\left(1-\frac{q_{1}+t_{1}}{q_{2}+t_{2}}\right) X_{2}, \\
& Z_{\mathbf{Y}}=\frac{1}{2}\left(1+\frac{q_{1}+t_{1}}{q_{2}+t_{2}}\right) Y_{1}+\frac{1}{2}\left(1-\frac{q_{1}+t_{1}}{q_{2}+t_{2}}\right) Y_{2},
\end{aligned}
$$

and the Corollary 3.15 with $\mathbf{a}=\frac{\|(Q+T) \mathbf{1}\|}{2 \sqrt{2}\left(q_{2}+t_{2}\right)}[1,1]^{\prime}$, we have that $Z_{\mathbf{Y}} \leq_{c v} Z_{\mathbf{X}}$. and $Z_{\mathbf{X}} \leq_{c x} Z_{\mathbf{Y}}$. Then a risk averse rational decision maker would prefer the portfolio $Z_{\mathbf{X}}$.

Moreover, the idea above allows also for criteria based on comparisons of either portfolio variances or risks measured through a convex measure in the sense of Föllmer and Schied [5]. Conditional Value at Risk (CVaR) is a convex risk measure; therefore under the same hypothesis as above, $Z_{\mathbf{X}}$ 
has less variance and smaller $(\mathrm{CVaR})$ than the portfolio $Z_{\mathbf{Y}}$ since variance and CVaR are consistent with respect to the convex order (see Shaked and Shanthikumar [24] pages 110 to 112 and Bäuerle and Mülle [2]).

Consider now the case in which the agent has to allocate his capital in two different but not independent risky assets $X_{1}$ and $X_{2}$. A typical problem in portfolio theory is the determination of the allocation $\alpha \in[0,1]$ such that $Z_{\alpha}=(1-\alpha) X_{1}+\alpha X_{2}$ maximizes the expected utility $h(\alpha)=E\left[U\left(Z_{\alpha}\right)\right]$, where $U$ is the increasing and concave utility function of the agent. In 1971, Hadar and Russel proved that, if $X_{1}$ and $X_{2}$ are iid, then $h\left(\frac{1}{2}\right) \geq h(\alpha)$ for all $\alpha \in[0,1]$, thus proving that the maximal diversification gives the maximal expected utility under the assumptions above. This result was generalized in Ma [15] to the multivariate case, replacing the assumption of independence with the assumption of exchangeability. Related results have been provided in Pellerey and Semeraro [20]. Specifically, they proved that if the vector $(S, D)$ of the sum $S=X_{1}+X_{2}$ and the difference $D=X_{2}-X_{1}$ of the risks is positive quadrant dependent (PQD), i.e., if $(S, D) \geq_{P Q D}\left(S^{\perp}, D^{\perp}\right)$ where $\left(S^{\perp}, D^{\perp}\right)$ is the independent version of $(S, D)$, and if $E\left[X_{2}\right] \leq E\left[X_{1}\right]$, then $h(\alpha)$ is decreasing in $\alpha=\left[\frac{1}{2}, 1\right]$. Similarly, they proved that if the vector $(S, D)$ is negative quadrant dependent, $N Q D$, (i.e., if $(S, D) \leq_{P Q D}\left(S^{\perp}, D^{\perp}\right)$ ) and $E\left[X_{2}\right] \geq E\left[X_{1}\right]$, then $h(\alpha)$ is increasing in $\alpha=\left[0, \frac{1}{2}\right]$. As a consequence of these results we have that if the vector $\left(X_{1}, X_{2}\right)$ is such that $E\left[X_{2}\right]=E\left[X_{1}\right]$, and $S$ and $D$ are uncorrelated, then $h\left(\frac{1}{2}\right) \geq h(\alpha)$ for all $\alpha \in[0,1]$. A generalization of this result is given here.

Theorem 4.3. Let $\mathbf{X}=\left(X_{1}, X_{2}\right)$ be random vector. Consider $\mathbf{u}=\left[u_{1}, u_{2}\right]^{\prime}$ a unit vector and $\mathbf{v}=\frac{\sqrt{2}}{2}\left[u_{1}-u_{2}, u_{1}+u_{2}\right]^{\prime}$. Let $\mathbf{Z}=\left(Z_{1}, Z_{2}\right)$ be any random 
vector of independent components with mean $\mathcal{R}_{\mathbf{v}} E[\mathbf{X}]$ and let $\mathbf{Y}=\mathcal{R}_{\mathbf{v}}^{\prime} \mathbf{Z}$. If $\mathbf{Y} \leq_{E_{\mathbf{v}}}\left[\geq_{E_{\mathbf{v}}}\right] \mathbf{X}$ and $u_{1} E\left[X_{2}\right] \leq[\geq] u_{2} E\left[X_{1}\right]$, then for every increasing and concave utility function $U$ it holds that

$$
\begin{aligned}
& E\left[U\left(\frac{\sqrt{2}}{2} u_{1} X_{1}+\frac{\sqrt{2}}{2} u_{2} X_{2}\right)\right] \\
& \geq E\left[U\left(\frac{\sqrt{2}}{2}\left(u_{1}+u_{2}-2 u_{2} \alpha\right) X_{1}+\frac{\sqrt{2}}{2}\left(2 u_{1} \alpha-u_{1}+u_{2}\right) X_{2}\right)\right]
\end{aligned}
$$

for all $\alpha \in\left[\frac{1}{2}, 1\right]$ [for all $\left.\alpha \in\left[0, \frac{1}{2}\right]\right]$.

Proof. We see the proof the case $\leq$, the another case is similar. It is immediate that $E[\mathbf{Y}]=E[\mathbf{X}]$. From Theorem 3.14 we deduce that $\mathcal{R}_{\mathbf{v}} \mathbf{Y} \leq_{P Q D} \mathcal{R}_{\mathbf{v}} \mathbf{X}$ since $\mathbf{Y} \leq_{E_{\mathbf{v}}} \mathbf{X}$. However, $\mathcal{R}_{\mathbf{v}} \mathbf{Y}$ has independent components, so $\mathcal{R}_{\mathbf{v}} \mathbf{X}$ is PQD. On account of (3.1) we obtain

$$
\left(\begin{array}{cc}
u_{1} & u_{2} \\
-u_{2} & u_{1}
\end{array}\right)\left(\begin{array}{c}
X_{1} \\
X_{2}
\end{array}\right) \quad \text { is PQD. }
$$

Using the formula (3.1) to construct $\mathcal{R}_{\mathbf{u}}$, with $\mathbf{u}=\left[u_{1}, u_{2}\right]^{\prime}$, we have that

$$
\mathcal{R}_{\mathbf{u}} \mathbf{X}=\frac{\sqrt{2}}{2}\left(\begin{array}{cc}
u_{1}+u_{2} & u_{2}-u_{1} \\
u_{1}-u_{2} & u_{1}+u_{2}
\end{array}\right)\left(\begin{array}{c}
X_{1} \\
X_{2}
\end{array}\right)
$$

Denote $X_{i}^{R}$ the $i$-th component of the vector $\mathcal{R}_{\mathbf{u}} \mathbf{X}, i=1,2$, and let $\left(S^{R}, D^{R}\right)^{\prime}$ be the vector of the sum and the difference of the components of $\left(X_{1}^{R}, X_{2}^{R}\right)^{\prime}$. Therefore, from (4.4),

$$
\begin{aligned}
& S^{R}=X_{1}^{R}+X_{2}^{R}=\sqrt{2} u_{1} X_{1}+\sqrt{2} u_{2} X_{2}, \\
& D^{R}=X_{2}^{R}-X_{1}^{R}=\sqrt{2} u_{1} X_{2}-\sqrt{2} u_{2} X_{1} .
\end{aligned}
$$


From (4.3) we have that $\left(S^{R}, D^{R}\right)^{\prime}$ is PQD. Since $u_{1} E\left[X_{2}\right] \leq u_{2} E\left[X_{1}\right]$, it follows that $E\left[X_{2}^{R}\right] \leq E\left[X_{1}^{R}\right]$. By using Theorem 2.1 in Pellerey and Semeraro [20], we get that

$Z_{\alpha}=(1-\alpha) X_{1}^{R}+\alpha X_{2}^{R}=\frac{\sqrt{2}}{2}\left(u_{1}+u_{2}-2 u_{2} \alpha\right) X_{1}+\frac{\sqrt{2}}{2}\left(2 u_{1} \alpha-u_{1}+u_{2}\right) X_{2}$

is decreasing in the concave order in $\alpha \in\left[\frac{1}{2}, 1\right]$. Thus, in particular, for every increasing and concave utility function $U$ it holds

$$
\begin{aligned}
& E\left[U\left(\frac{\sqrt{2}}{2} u_{1} X_{1}+\frac{\sqrt{2}}{2} u_{2} X_{2}\right)\right] \\
& \geq E\left[U\left(\frac{\sqrt{2}}{2}\left(u_{1}+u_{2}-2 u_{2} \alpha\right) X_{1}+\frac{\sqrt{2}}{2}\left(2 u_{1} \alpha-u_{1}+u_{2}\right) X_{2}\right)\right]
\end{aligned}
$$

for all $\alpha \in\left[\frac{1}{2}, 1\right]$.

Remark 4.4. Note that if there exists a vector $u=\left(u_{1}, u_{2}\right)$ such that

$$
\left(\begin{array}{cc}
u_{1} & u_{2} \\
-u_{2} & u_{1}
\end{array}\right)\left(\begin{array}{c}
X_{1} \\
X_{2}
\end{array}\right)
$$

has uncorrelated components (so that it is neither PQD nor NQD), then the maximal expected utility is reached in

$$
Z_{1 / 2}=\frac{\sqrt{2}}{2} u_{1} X_{1}+\frac{\sqrt{2}}{2} u_{2} X_{2}
$$

Since $\frac{\sqrt{2}}{2} u_{1}+\frac{\sqrt{2}}{2} u_{2} \leq 1$, this means that under such conditions it is not necessary to invest totally the available capital. It also is interesting to observe that some of the allocations can be negative, thus the case of short selling are allowed. 


\section{Optimal portfolio selection through rotations}

We now consider the particular case that the risks have joint elliptical distributions that is a common assumption in portfolio theory since they allow for the presence of heavy tails and asymptotic tail dependence distributions. The importance and applications of elliptical distributions for risk management and insurance have been widely studied by Embrechts et al. [4], Landsman [11] and Landsman and Valdez [10].

Definition 5.1. The random vector $\mathbf{X}=\left(X_{1}, \ldots, X_{n}\right)^{\prime}$ is said to have an elliptical distribution with parameters $\mu$ and $\Sigma$ if its characteristic function can be expressed as

$$
E\left[\exp \left(i \mathbf{t}^{\prime} X\right)\right]=\exp \left(i \mathbf{t}^{\prime} \mu\right) \phi\left(\mathbf{t}^{\prime} \boldsymbol{\Sigma} \mathbf{t}\right), \quad \mathbf{t}=\left(t_{1}, \ldots, t_{n}\right)^{\prime}
$$

for some function $\phi$, and if $\boldsymbol{\Sigma}$ is such that $\mathbf{\Sigma}=\mathbf{A} \mathbf{A}^{\prime}$ for some matrix $\mathbf{A}(n \times$ $m)$.

The moments of $\mathbf{X}$ do not necessarily exist. However, if the mean vector exits, then it is the parameter $\mu$. Besides, if the covariance matrix exists, then it is given by $\operatorname{Cov}(\mathbf{X})=-2 \phi^{\prime}(0) \Sigma$. A necessary condition for this covariance matrix to exist is $\left\|\phi^{\prime}(0)\right\|<\infty$, where $\phi^{\prime}$ denotes the first derivative of the characteristic generator $\phi$ of $\mathbf{X}$. Note that the class of multivariate elliptical distribution with $\phi(x)=\exp \left(-\frac{x}{2}\right)$ corresponds to the class of multivariate normal distribution.

Recall that the components of a random vector $\mathbf{X}=\left(X_{1}, \ldots, X_{n}\right)^{\prime}$ are said to be exchangeable if for any permutation matrix $\mathbf{P}$ the vector $\mathbf{P X}$ has the same distribution as $\mathbf{X}$. The following property will be used later. 
Property 5.2. Let $\mathbf{X}=\left(X_{1}, X_{2}\right)$ be a random vector elliptically distributed with parameters $\mu=\mathbf{0}$ and $\mathbf{\Sigma}_{\mathbf{X}}$. Then there exists a unit vector $\mathbf{u}$ such that $\mathcal{R}_{\mathbf{u}} \mathbf{X}$ is exchangeable.

Proof. Since $\boldsymbol{\Sigma}_{\mathbf{X}}$ is a symmetric matrix, then it can be expressed as $\boldsymbol{\Sigma}_{\mathbf{X}}=$ $Q D Q^{\prime}$, where $Q=\left(q_{i j}\right)$ is an orthogonal matrix and $D=\left(d_{i i}\right)$ is a diagonal matrix with non-negative elements. Let $\mathbf{u}=\left(q_{11}, q_{21}\right)^{\prime}$ be the first column of the matrix $Q$. Then, according to (3.1),

$$
\mathcal{R}_{\mathbf{u}}=\frac{\sqrt{2}}{2}\left(\begin{array}{ll}
q_{11}+q_{21} & q_{21}-q_{11} \\
q_{11}-q_{21} & q_{11}+q_{21}
\end{array}\right) .
$$

The vector $\mathcal{R}_{\mathbf{u}} \mathbf{X}$ also is elliptically distributed, with parameters $\mathcal{R}_{\mathbf{u}} \mu=\mathbf{0}$ and $\boldsymbol{\Sigma}_{\mathcal{R}_{\mathbf{u}} \mathbf{X}}=\mathcal{R}_{\mathbf{u}} \Sigma_{\mathbf{X}} \mathcal{R}_{\mathbf{u}}^{\prime}$ (see, e.g., Valdez and Dhaene [25], Theorem 2).

It is clear that

$$
\boldsymbol{\Sigma}_{\mathcal{R}_{\mathbf{u}} \mathbf{X}}=\mathcal{R}_{\mathbf{u}} Q D Q^{\prime} \mathcal{R}_{\mathbf{u}}^{\prime}=\frac{1}{2}\left(\begin{array}{ll}
d_{11}+d_{22} & d_{11}-d_{22} \\
d_{11}-d_{22} & d_{11}+d_{22}
\end{array}\right) .
$$

Observe that $\boldsymbol{\Sigma}_{\mathcal{R}_{\mathrm{u}} \mathrm{X}}$ is symmetric, with the same diagonal elements, which implies that for any permutation matrix $\mathbf{P}$ we have that $\mathbf{P}^{\prime} \boldsymbol{\Sigma}_{\mathcal{R}_{\mathrm{u}} \mathrm{X}} \mathbf{P}=\boldsymbol{\Sigma}_{\mathcal{R}_{\mathrm{u}} \mathrm{x}}$. It is easily seen that $\mathbf{P}^{\prime} \mathcal{R}_{\mathbf{u}} \mathbf{X}$ is elliptically distributed. Hence, its characteristic function will be given by

$$
E\left[\exp \left(i \mathbf{t}^{\prime} X\right)\right]=\phi\left(\mathbf{t}^{\prime} \mathbf{P}^{\prime} \boldsymbol{\Sigma}_{\mathcal{R}_{\mathbf{u}} \mathbf{X}} \mathbf{P t}\right)=\phi\left(\mathbf{t}^{\prime} \boldsymbol{\Sigma}_{\mathcal{R}_{\mathbf{u}} \mathbf{X}} \mathbf{t}\right), \quad \mathbf{t}=\left(t_{1}, t_{2}\right)^{\prime} .
$$

Since the characteristic function determines the distribution, then by (5.2) we have that $\mathcal{R}_{\mathbf{u}} \mathbf{X}={ }_{\text {st }} \mathbf{P} \mathcal{R}_{\mathbf{u}} \mathbf{X}$, and as a consequence, the vector $\mathcal{R}_{\mathbf{u}} \mathbf{X}$ has exchangeable components. 
The next statement describes conditions to compare in the concave order two different portfolios of elliptically distributed risky assets, with possibility of negative weights.

Theorem 5.3. Let $\mathbf{X}=\left(X_{1}, X_{2}\right)$ be a elliptically distributed random vector with parameter $\boldsymbol{\Sigma}$ and vector of means $\mu=\mathbf{0}$. Let $\mathbf{u}=\left(u_{1}, u_{2}\right)^{\prime}$ be an eigenvector of $\boldsymbol{\Sigma}$. If $\left(\alpha_{1}, \alpha_{2}\right)$ and $\left(\beta_{1}, \beta_{2}\right)$ are such that $\alpha_{1}+\alpha_{2}=\beta_{1}+\beta_{2}$ and $\alpha_{1} \leq \beta_{1}$, then

$$
\begin{aligned}
& \frac{\sqrt{2}}{2}\left[\alpha_{1}\left(u_{1}+u_{2}\right)+\alpha_{2}\left(u_{2}-u_{1}\right)\right] X_{1}+\frac{\sqrt{2}}{2}\left[\left(\alpha_{1}\left(u_{1}-u_{2}\right)+\alpha_{2}\left(u_{1}+u_{2}\right)\right] X_{2}\right. \\
& \geq_{c v} \frac{\sqrt{2}}{2}\left[\beta_{1}\left(u_{1}+u_{2}\right)+\beta_{2}\left(u_{2}-u_{1}\right)\right] X_{1}+\frac{\sqrt{2}}{2}\left[\left(\beta_{1}\left(u_{1}-u_{2}\right)+\beta_{2}\left(u_{1}+u_{2}\right)\right] X_{2} .\right.
\end{aligned}
$$

Proof. As $\mathbf{u}=\left(u_{1}, u_{2}\right)^{\prime}$ is a eigenvector of $\boldsymbol{\Sigma}$, we deduce from Property 5.2 that

$$
\mathcal{R}_{\mathbf{u}} \mathbf{X}=\frac{\sqrt{2}}{2}\left(\begin{array}{cc}
u_{1}+u_{2} & u_{2}-u_{1} \\
u_{1}-u_{2} & u_{1}+u_{2}
\end{array}\right)\left(\begin{array}{c}
X_{1} \\
X_{2}
\end{array}\right)
$$

is exchangeable. Applying Theorem 3.A.35 in Shaked and Shanthikumar [24] we conclude the proof.

The following Property is an extension of the Property 5.2 for highdimension

Property 5.4. Let $\mathbf{X}=\left(X_{1}, \ldots, X_{n}\right)^{\prime}$ be a random vector elliptically distributed with parameters $\mu_{\mathbf{X}}=0$ and $\boldsymbol{\Sigma}_{\mathbf{X}}$ is such that it has at least $n-1$ equal eigenvalues given by $\lambda_{1} \geq \lambda_{2}=\cdots=\lambda_{n}=\lambda>0$. Then there exists a unit vector $\mathbf{u}$ such that $\mathcal{R}_{\mathbf{u}} \mathbf{X}$ has exchangeable components .

Proof. Our proof starts with the observation that the singular values decomposition (SVD) of $\boldsymbol{\Sigma}_{\mathbf{X}}$ is given by $\boldsymbol{\Sigma}_{\mathbf{X}}=Q D Q^{\prime}$, where $D=\operatorname{diag}\left\{\lambda_{1}, \lambda, \ldots, \lambda\right\}$ 
and $Q=\left(q_{i j}\right)$ is an orthogonal matrix. Consider the unit vector $\mathbf{u}=Q^{(c .1)}=$ $\left[q_{11}, q_{21}, \ldots, q_{n 1}\right]^{\prime}$. From (3.1) we have that $\mathcal{R}_{\mathbf{u}} Q^{(c .1)}=\frac{1}{\sqrt{n}}[1, \ldots, 1]^{\prime}$. It is easy to check that

$$
\boldsymbol{\Sigma}_{\mathcal{R}_{\mathbf{u}} \mathbf{x}}=\mathcal{R}_{\mathbf{u}} Q D Q^{\prime} \mathcal{R}_{\mathbf{u}}^{\prime}=\left(\mathcal{R}_{\mathbf{u}} Q \sqrt{D}\right)\left(\mathcal{R}_{\mathbf{u}} Q \sqrt{D}\right)^{\prime}
$$

Taking $H=\mathcal{R}_{\mathbf{u}} Q$ we can rewrite $(5.3)$ as $(H \sqrt{D})(H \sqrt{D})^{\prime}$. Of course, $H$ is an orthogonal matrix whose first column is $\frac{1}{\sqrt{n}}[1, \ldots, 1]^{\prime}$. Let $\sigma_{i j}^{*}$ be the element $(i j)$ of the matrix (5.3). Hence,

$$
\begin{aligned}
\sigma_{i j}^{*} & =\frac{\lambda_{1}}{n}+\lambda \sum_{j=2}^{n} h_{i j}^{2}=\frac{\lambda_{1}}{n}+\lambda\left(1-\frac{1}{n}\right)=\frac{\lambda_{1}+\lambda(n-1)}{n} \\
& =\frac{\operatorname{tr}\left(\boldsymbol{\Sigma}_{\mathbf{X}}\right)}{n}=\frac{1}{n} \sum_{i=1}^{n} \operatorname{var}\left(X_{i}\right), \quad \text { if } \quad i=j . \\
\sigma_{i j}^{*} & =\frac{\lambda_{1}}{n}+\lambda \sum_{k=2}^{n} h_{i k} h_{j k}=\frac{\lambda_{1}}{n}+\lambda\left(-\frac{1}{n}\right)=\frac{\lambda_{1}-\lambda}{n}, \quad \text { if } \quad i \neq j .
\end{aligned}
$$

It is clear that the diagonal of $\boldsymbol{\Sigma}_{\mathcal{R}_{\mathrm{u}} \mathrm{X}}$ has the same elements and the offdiagonal also have the same elements. It follows that $P^{\prime} \boldsymbol{\Sigma}_{\mathcal{R}_{\mathrm{u}} \mathbf{x}} P=\boldsymbol{\Sigma}_{\mathcal{R}_{\mathrm{u}} \mathbf{X}}$ for any $(n \times n)$ permutation matrix $\mathbf{P}$. It is easily seen that $\mathbf{P}^{\prime} \mathcal{R}_{\mathbf{u}} \mathbf{X}$ is elliptically distributed (see, e.g., Valdez and Dhaene [25], Theorem 2). Hence, its characteristic function will be given by

$$
E\left[\exp \left(i \mathbf{t}^{\prime} X\right)\right]=\phi\left(\mathbf{t}^{\prime} \mathbf{P}^{\prime} \boldsymbol{\Sigma}_{\mathcal{R}_{\mathbf{u}} \mathbf{x}} \mathbf{P t}\right)=\phi\left(\mathbf{t}^{\prime} \boldsymbol{\Sigma}_{\mathcal{R}_{\mathbf{u}} \mathbf{x}} \mathbf{t}\right), \quad \mathbf{t}=\left(t_{1}, \ldots, t_{n}\right)^{\prime} .
$$

By the one-to-one correspondence between distribution functions and characteristic functions, and from (5.4), $\mathcal{R}_{\mathbf{u}} \mathbf{X}={ }_{s t} \mathbf{P} \mathcal{R}_{\mathbf{u}} \mathbf{X}$, and as a consequence the vector $\mathcal{R}_{\mathbf{u}} \mathbf{X}$, has exchangeable components. 
Remark 5.5. Property 5.4 is also valid when $\mu_{\mathbf{X}}=k Q^{(c .1)}$ for some $k \in \mathbb{R}$ since $\mathcal{R}_{\mathbf{u}} \mu_{\mathbf{X}}=k \mathcal{R}_{\mathbf{u}} Q^{(c .1)}=\frac{k}{\sqrt{n}}[1, \ldots, 1]^{\prime}$. Therefore, $\mathcal{R}_{\mathbf{u}} \mathbf{X}$ has exchangeable components. In Theorem 5.4 obviously $k=0$.

We are thus led to the following strengthening of Theorem 5.3

Theorem 5.6. Let $\mathbf{X}=\left(X_{1}, \ldots, X_{n}\right)^{\prime}$ satisfy the hypotheses of the Property 5.4. Suppose that the (SVD) of $\boldsymbol{\Sigma}_{\mathbf{X}}$ is given by $\boldsymbol{\Sigma}_{\mathbf{X}}=Q D Q^{\prime}$ and let $\mathbf{u}=Q^{(c .1)}$ be the unit vector. If $\mathbf{a}=\left(a_{1}, \ldots, a_{n}\right)^{\prime}$ is majorized by $\mathbf{b}=\left(b_{1}, \ldots, b_{n}\right)^{\prime}$, then

$$
\sum_{i=1}^{n} \mathbf{a}^{\prime} \mathcal{R}_{\mathbf{u}}^{(c . i)} X_{i} \geq_{c v} \sum_{i=1}^{n} \mathbf{b}^{\prime} \mathcal{R}_{\mathbf{u}}^{(c . i)} X_{i} .
$$

Proof. We conclude from Property 5.4 that $\mathcal{R}_{\mathbf{u}} \mathbf{X}$ has exchangeable components; hence the assertion follows by Theorem 3.A.35 in Shaked and Shanthikumar [24].

\section{Conclusions}

We have introduced in this paper a generalization of the upper and lower orthant orders. This new stochastic order allows for comparisons of random vectors in different directions. We also have given some properties and their relationships with other stochastic orders studied in the literature. From applications point of view, we consider the single period portfolio problem of allocating the wealth to $n$ risks. Some solutions to this problem are given when two random vectors are comparable in extremality order sense. In the special case of risks elliptically distributed, we have studied directions to rotate the distributions and finding easily the optimal allocations of the wealth in order to maximize the expected utility of a risk averse decision maker. 


\section{Acknowledgements}

This research has been supported by projects of the Spanish Ministry of Science and innovation (2009/00035/001 and SEJ2007-64500), by the COMUNIDAD DE MADRID-UC3M project (2011/00068/001), and by the Italian PRIN 2008 project "Probabilitá e Finanza". The first author thanks the support given by the OTRI-UC3M and the hospitality of the Mathematical Department of the Politecnico di Torino.

\section{References}

[1] Barlow, R.E., Proshan, F., 1975. Statistical theory of reliability and life testing:probability models hold. Rinehart and Winston, New York

[2] Bäuerle, N., Müller, A., 2006. Stochastic orders and risk measures: consistency and bounds. Insurance: Mathematics \& Economics 38, 132-148.

[3] Denuit, M., Dhaene, J., Goovaerts, M., Kaas, R., 2005. Actuarial theory for dependent risks: measures, orders and models. Wiley, Chichester.

[4] Embrechts, P., McNeil, A., Straumann, D., 2002. Correlation and dependence in risk management: properties and pitfalls. in Management: Values at Risk and Beyond, Dempster, M.,Moffatt, H.K. (Eds.) Cambridge University Press, pp. 176-223.

[5] Föllmer, H., Schied, A., 2002. Convex measures of risk and trading constraints. Finance and Stochastic 6, 429-447.

[6] Hadar, J., Russel, W.R., 1971. Stochastic dominance and diversification. Journal of Economic Theory 3, 288-305. 
[7] Jorion, P., 1997. Value at Risk: the new benchmark for controlling market risk. Irwin, Chicago.

[8] Karlin, S., Rinott, Y., 1980. Classes of orderings of measures and related correlation inequalities, I. Multivariate totally positive distribution. Journal of Multivariate Analysis 10, 467-498.

[9] Karlin, S., Rinott, Y., 1981. Total positivity properties of absolute value multinormal variables with applications to confidence interval estimates and related probabilistic inequalities. The Annals of Statistics. 9, 10351049 .

[10] Landsman, Z., Valdez, E., 2003. Tail conditional expectations for elliptical distributions. North American Actuarial Journal 7, 4 55-71.

[11] Landsman, Z., 2004. On the generalization of Esscher and variance premiums modified for the elliptical family of distributions. Insurance: Mathematics \& Economics 35, 563-579.

[12] Landsman, Z., Tsanakas, A., 2006. Stochastic ordering of bivariate elliptical distributions. Statistics \& Probability Letters 76, 488-494.

[13] Laniado, H., Lillo, R.E., Romo, J., 2010. Multivariate extremality measure. Working paper. Available at http://e-archivo.uc3m.es/bitstream/10016/8970/1/ws101908.pdf

[14] Lehmann, E.L., 1966. Some concepts of dependence. Annals of Mathematical Statistics 37, 1137-1153. 
[15] Ma, C., 2000. Convex order for linear combinations of random variables. Journal of Statistical Planning and Inference 84, 11-25.

[16] Markowitz, H. M., 1952. Mean-variance analysis in portfolio choice and capital markets. Journal of Finance 7, 77-91.

[17] Marshall, A.W., Olkin, I., 1979. Inequalities: theory of majorization and its applications. New York: Academic Press.

[18] Müller, A., Stoyan, D., 2002. Comparison methods for stochastic models and risk. Wiley, New York.

[19] Nelsen , R.B., 1999 An introduction to copulas. Springer-Verlang, New York.

[20] Pellerey, F., Semeraro, P., 2005. A note on the portfolio selection problem. Theory and Decision 59, 295-306.

[21] Rinott, Y., Scarsini, M., 2006. Total positivity order and the normal distribution. Journal of Multivariate Analysis 97, 1251-1261.

[22] Scarsini, M., 1988. Multivariate stochastic dominance with fixed dependence structure. Operations Research Letters 7, 237-240.

[23] Stoyan, D., 1983. Comparison methods for queues and other stochastic models. Wiley, New York.

[24] Shaked, M., Shanthikumar, J.G., 2007. Stochastic orders. Springer, New York. 
[25] Valdez, E.A. and Dhaene, J., 2004. Convex order bounds for sum of dependent log-elliptical random variables. 7th International Congress on Insurance: Mathematics \& Economics, Lyon, France, June 25-27.

[26] Yaari, M.E., 1987. The dual theory of choice inder risk. Econometrica $55,95-115$. 